\title{
Recruiting participants to walking intervention studies: a systematic review
}

Charlie E Foster $^{1 *}$, Graham Brennan ${ }^{2}$, Anne Matthews ${ }^{1}$, Chloe McAdam², Claire Fitzsimons ${ }^{2}$ and Nanette Mutrie ${ }^{2}$

\begin{abstract}
Purpose: Most researchers who are conducting physical activity trials face difficulties in recruiting participants who are representative of the population or from specific population groups. Participants who are often the hardest to recruit are often those who stand to benefit most (the least active, from ethnic and other minority groups, from neighbourhoods with high levels of deprivation, or have poor health). The aim of our study was to conduct a systematic review of published literature of walking interventions, in order to identify the impact, characteristics, and differential effects of recruitment strategies among particular population groups.

Methods: We conducted standard searches for studies from four sources, (i) electronic literature databases and websites, (ii) grey literature from internet sources, (iii) contact with experts to identify additional "grey" and other literature, and (iv) snowballing from reference lists of retrieved articles. Included studies were randomised controlled trials, controlled before-and-after experimental or observational qualitative studies, examining the effects of an intervention to encourage people to walk independently or in a group setting, and detailing methods of recruitment.

Results: Forty seven studies met the inclusion criteria. The overall quality of the descriptions of recruitment in the studies was poor with little detail reported on who undertook recruitment, or how long was spent planning/ preparing and implementing the recruitment phase. Recruitment was conducted at locations that either matched where the intervention was delivered, or where the potential participants were asked to attend for the screening and signing up process. We identified a lack of conceptual clarity about the recruitment process and no standard metric to evaluate the effectiveness of recruitment.

Conclusion: Recruitment concepts, methods, and reporting in walking intervention trials are poorly developed, adding to other limitations in the literature, such as limited generalisability. The lack of understanding of optimal and equitable recruitment strategies evident from this review limits the impact of interventions to promote walking to particular social groups. To improve the delivery of walking interventions to groups which can benefit most, specific attention to developing and evaluating targeted recruitment approaches is recommended.
\end{abstract}

Keywords: Recruitment, walking, physical activity, health promotion

\section{Introduction}

It is over a decade since Professors Jerry Morris and Adrienne Hardman described walking as the 'nearest activity to perfect exercise' (Hardman \& Morris, p328, 1997) [1]. The epidemiological research underpinning their statement has rapidly increased, so that the promotion of walking is now a central pillar in many international physical activity strategies and national plans, e.

\footnotetext{
* Correspondence: charlie.foster@dph.ox.ac.uk

'Department of Public Health, University of Oxford, UK

Full list of author information is available at the end of the article
}

g. 2010 Toronto Charter for Physical Activity [2]. Regular walking, independent of other physical activity, can reduce the risk of overall mortality, of cardiovascular disease (CVD) and improve risk factors for CVD, including diastolic blood pressure and lipid profiles [3-5]. Regular walking is associated with a reduction in body mass index and body weight, with reduced risk of type 2 diabetes [6] and is suggested to improve self esteem, relieve symptoms of depression and anxiety, and improve mood $[7,8]$. From a public health perspective, enabling an increase in overall population levels of

\section{Ciomed Central}


physical activity through walking will produce an effective reduction in risk of all cause mortality [9].

A systematic review of the effectiveness of walking interventions found evidence for a range of approaches [10]. These included brief advice to individuals, remote support to individuals, group-based approaches, active travel and community level approaches. Recent reviews have provided evidence to support environmental and school based travel interventions [10-12]. Despite the evidence for the benefits of walking for health, population rates of walking and overall physical activity remain low and below recommended levels [13-15]. Population surveys report that walking behaviour is socially patterned by gender, age, socio-economic status (SES) and by the purpose of walking i.e. for leisure or transport. For example, in the UK long brisk paced walks are more common among affluent groups, whereas walking for transport is more common among less affluent groups $[14,16]$.

One criticism of the evidence base for walking interventions is a failure to recruit specific groups of the population and further studies are needed to broaden the reach of walking interventions [10-12]. Intervention reach, or recruiting specific population sub-groups, is only partially reflected in public health and clinical research. For example the RE-AIM framework is designed to guide the implementation of behaviour change interventions [17]. It recommends assessing both an intervention's effectiveness and ability to reach a targeted group. Similarly, recent CONSORT (2010) guidelines [18] recommend clearly displaying the flow of participants throughout a study. Despite identifying recruitment as part of their framework, the guidelines do not define the actions needed to identify and recruit potential populations of participants. There is an absence of conceptual frameworks for recruitment to intervention studies and also a lack of procedural models and systems for recruitment. There is a need to identify what factors are effective in engaging participation at the recruitment phase [19-21].

Research examining recruitment practice has focused on drug or medical interventions rather than public health interventions [22]. Little is known about recruitment to physical activity interventions. A Cochrane review identified three stages of recruitment (invitation, screening, intervention starting) for potential participants into physical activity randomised control trials (RCTs). The authors noted a considerable loss of participants across each stage limiting the effectiveness of interventions [23]. The CONSORT (2010) guidelines, suggest that studies report the number of eligible participants prior to randomisation but do not insist on the need to report the original overall number of responders invited to participate (prior to eligibility) [18].
Clearly the effectiveness of a walking programme is limited by not only its efficacy of dose (how well the intervention works on its participants) but also by its recruitment (maximising the numbers who will participate and receive the intervention dose). In response to frequent research calls to evaluate effective approaches to the recruitment of individuals to walking studies, the Scottish Physical Activity Research Collaboration http:// www.sparcoll.org.uk undertook a series of studies to examine recruitment strategies for research and community based programmes of walking promotion. We defined recruitment for such walking studies or programmes as the process of inviting participation to a formal activity including the invitation, informing and facilitation of interested parties to take part in an organised study, activity or event. This paper reports the results of a systematic review to examine the reported recruitment procedures of walking studies, in order to identify the characteristics of effective recruitment, and the impact and differential effects of recruitment strategies among particular population groups.

\section{Method}

\section{Identification of studies}

We used The Quality of Reporting of Meta-analysis statement (QUOROM) to provide the structure for our review [24]. We identified four possible sources of potential studies, (i) electronic literature databases and websites, (ii) grey literature from internet sources, (iii) contact with experts to identify additional "grey" and other literature, and (iv) snowballing from reference lists of retrieved articles. In the first stage of the literature search, titles and abstracts of identified articles were checked for relevance. In the second stage, full-text articles were retrieved and considered for inclusion. In the final stage, the reference lists of retrieved full-text articles were searched and additional articles known to the authors were assessed for possible inclusion. We conducted a systematic search of electronic databases including OVID MEDLINE, EMBASE, PsychINFO, PubMed, Scopus, SIGLE and SPORTDiscus. We searched a number of web based databases including National Institute of Health and Clinical Excellence (NICE), Effective Public Health Project (EPHP Hamilton), Health Evidence Canada, and the Evidence for Policy and Practice Information and Co-ordinating Centre (EPPI)). We conducted searches of internet sites of key international walking promotion agencies including Walk England, the Centers for Disease Control and Prevention $(\mathrm{CDC})$ and the World Health Organisation (WHO).

Studies published from the end of 2000 up to and including the search date $(05 / 2009)$ were considered for inclusion. Individualized search strategies for the 
different databases included combinations of the following key words: (walk*) AND (recruit* OR participat* OR market*). Articles published or accepted for publication in refereed journals were considered for the review. Articles reported in UK grey and web based literature including any evidence of types of recruitment approaches and strategies, any evidence of effectiveness, economic costs, and evidence of any differential response to recruitment approaches were also considered in the review. Conference proceedings and abstracts were included if further searching of the databases or contact with the author was able to retrieve a full article from the study presented in the original piece of literature. We sent emails to international experts, identified in a previous systematic review on walking promotion [10].

\section{Criteria for study inclusion/exclusion}

Titles, abstracts and reports were independently assessed (by AM, CF and GB) for inclusion. Studies were considered to be eligible for inclusion according to the following criteria: (i) participants were of any age and were not trained athletes or sports students, (ii) studies of any type including randomised controlled trials, controlled before-and-after experimental or observational studies, (iii) studies that examined the effects of an intervention to encourage people to walk independently or in a group setting, (iv) interventions of any kind and in any field, whether targeted on individuals, communities, settings, groups or whole populations, (v) details of methods of recruitment were reported or were retrievable through correspondence with the authors, (vi) qualitative studies that examined the experiences of the participants during recruitment and which aimed to assess the effectiveness of the recruitment methods used, and (vii) studies published in English.

Included studies were categorised by study design using standardised criteria for quantitative experimental or observational studies (e.g. RCT, non-Randomised Control Trials (NRCT), before-and-after, cross-sectional), or qualitative studies (e.g. focus groups) [25].

\section{Criteria for assessment of study quality in relation to recruitment}

Two authors (GB and CF) independently assessed the quality of the studies in relation to recruitment description that met the inclusion criteria. The criteria for assessing the recruitment reporting quality of each study were adapted from Jadad (1998) [26], and in consultation with experts. A formal quality score for each study was completed on a 5 -point scale by assigning a value of 0 (absent or inadequately described) or 1 (explicitly described and present) to each of the following questions listed: (i) did the study report where the population was recruited? (ii) did the study report who conducted the recruitment? (iii) did the study report the time spent planning/preparing the recruitment? (iv) did the study report the time spent conducting the recruitment? (v) did the study target a specific population? Studies that scored 4-5 were considered as high quality studies while studies that scored 1-3 were considered low quality.

\section{Criteria for assessing efficiency and effectiveness}

Where possible we calculated recruitment rates and efficiency ratios for each study, based on a previous systematic review of interventions to promote physical activity [23]. We defined four terms, (i) "pool"-the total number of potential participants who could be eligible for study, (ii) "invited"-the total number of potential participants invited to participate in the study, (iii) "responded"-the total number of potential participants who responded to the invitation, (iv) "started"-the number of participants who were assessed as eligible to participate and began the programme. If data were reported we calculated ratios for each stage, e.g. started/ pool-by dividing the number of participants who started into the study by the total reported in the pool, and expressed as proportions. If possible we attempted to calculate a weekly rate of recruitment for those studies on the number of weeks/months spent recruiting per participant.

\section{Results}

\section{Study Characteristics}

Fifty three papers representing 47 studies met our inclusion criteria. Duplicate studies were excluded and the journal article reporting the most recruitment data was analysed. The flow of studies through the review process is reported in Figure 1. Characteristics of included studies are presented in Table 1, ranked by quality score. Each included paper is referenced in the results and discussion sections in superscript, using their Study Number presented in Table 1. Full references for included papers are listed in additional file 1 and are presented in this paper in superscript form. Studies were located in the USA (24) [27-50], Australia (11) [51-61], UK (7) [62-68], Canada (3) [69-71], and one each from New Zealand [72] and Belgium [73]. Nearly all the studies were quantitative experimental studies in design, with twenty six randomised controlled trials, $[4,27,28,32-34,36-38,42,43,46,47,49,52$, $54,56,58,62-67,70]$ two studies reporting methods only $[28,35]$, three non-randomised controlled trials $[31,41,73]$ and seventeen before-and-after studies [27,29,30,39,40,44,45,48,50, 51,53,55,59-61,68,71] (two reporting methods only) $[27,30]$. We found only two qualitative studies reporting on recruitment approaches 


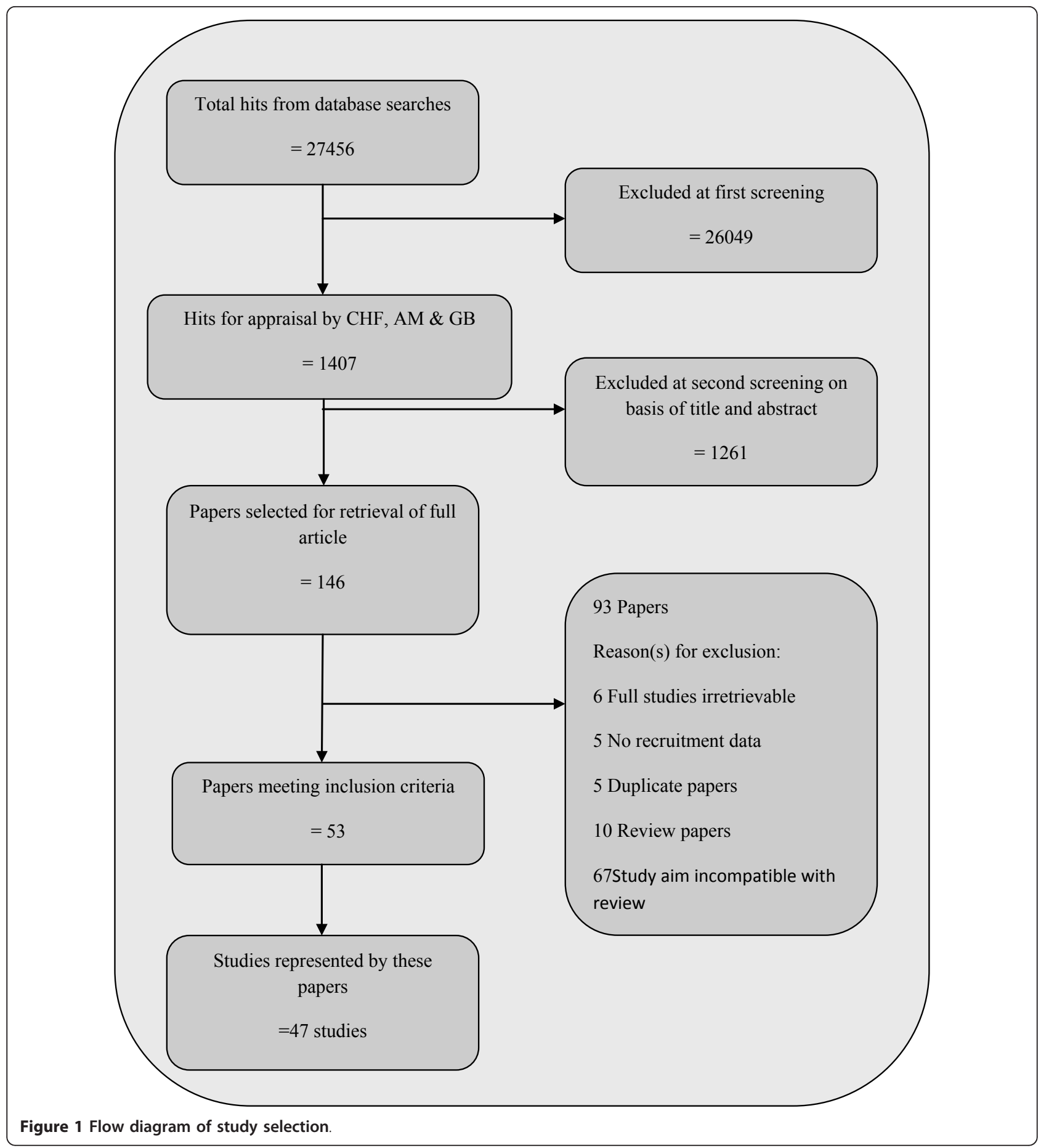

$[57,69]$, with one paper reporting qualitative data as part of an RCT study [64]. No studies were located from grey literature sources.

\section{Overview of study quality in relation to recruitment}

Eight studies were classified as "high" quality $[27-30,51,62,69,72]$ and the remaining thirty nine classified as "low" quality in relation to recruitment description
(Table 2-Assessment of study quality). Forty five studies reported a setting where the recruitment of participants took place [27-49,51-67,69-73] but only twenty two reported who conducted the recruitment [27-31,33,35-40,45,51-54,62,64,65,69,72]. Eleven studies reported the time spent conducting their recruitment $[27-30,32,51,62,63,66,70,72]$ three studies reported the time spent planning/preparing recruitment $[34,51,69]$. 
Table 1 Characteristics of included studies

\begin{tabular}{|c|c|c|c|c|c|}
\hline $\begin{array}{l}\text { Study Number, } \\
\text { Author and } \\
\text { Pub. Year }\end{array}$ & Country & $\begin{array}{l}\text { Study } \\
\text { Type }\end{array}$ & Study aim & Target Population & $\begin{array}{l}\text { Quality } \\
\text { Metric } \\
\text { Score }\end{array}$ \\
\hline $\begin{array}{l}\text { 1. Watson et al, } \\
2005\end{array}$ & Australia & $\begin{array}{l}\text { Before- } \\
\text { and-after } \\
\text { study }\end{array}$ & $\begin{array}{l}\text { Evaluate the effect of pram walking groups on self-reported } \\
\text { PA, mental health and social indicators. }\end{array}$ & Post-natal mothers & 5 \\
\hline $\begin{array}{l}\text { 2. Banks-Wallace } \\
\text { et al, } 2004\end{array}$ & USA & $\begin{array}{l}\text { Before- } \\
\text { and-after } \\
\text { study } \\
\text { Methods } \\
\text { paper }\end{array}$ & $\begin{array}{l}\text { Examine the effect of pre-intervention meetings as a strategy } \\
\text { for recruitment of African American women to a walking } \\
\text { programme. }\end{array}$ & $\begin{array}{l}\text { African American women in a } \\
\text { local community (Minority } \\
\text { group) }\end{array}$ & 4 \\
\hline 3. Kolt et al, 2006 & $\begin{array}{l}\text { New } \\
\text { Zealand }\end{array}$ & $\mathrm{RCT}$ & $\begin{array}{l}\text { To investigate the effectiveness of a telephone-based } \\
\text { counselling intervention aimed to increase physical activity in } \\
\text { sedentary older adults. }\end{array}$ & Older sedentary adults (> 65) & 4 \\
\hline $\begin{array}{l}\text { 4. Nguyen et al, } \\
2002\end{array}$ & Canada & Qualitative & $\begin{array}{l}\text { To evaluate the experience of delivering a walking club } \\
\text { (qualitative method) }\end{array}$ & General community & 4 \\
\hline $\begin{array}{l}\text { 5. Prestwich et } \\
\text { al, } 2010\end{array}$ & UK & $\mathrm{RCT}$ & $\begin{array}{l}\text { To test the effect of implementation intentions and text } \\
\text { messages on the promotion of brisk walking. }\end{array}$ & University students & 4 \\
\hline $\begin{array}{l}\text { 6. Rowland et al, } \\
2004\end{array}$ & USA & $\begin{array}{l}\text { RCT } \\
\text { Methods } \\
\text { paper }\end{array}$ & $\begin{array}{l}\text { To report on the recruitment of sedentary adults to the SHAPE } \\
\text { programme }\end{array}$ & Sedentary older adults & 4 \\
\hline $\begin{array}{l}\text { 7. Sherman et al, } \\
2006\end{array}$ & USA & $\begin{array}{l}\text { Before- } \\
\text { and-after } \\
\text { study }\end{array}$ & $\begin{array}{l}\text { Effect of a brief primary care based walking intervention in } \\
\text { rural women }\end{array}$ & Rural women & 4 \\
\hline $\begin{array}{l}\text { 8. Wilbur et al, } \\
2006\end{array}$ & USA & $\begin{array}{l}\text { Before- } \\
\text { and-after } \\
\text { study } \\
\text { Methods } \\
\text { paper }\end{array}$ & $\begin{array}{l}\text { To identify strategies successful in the recruitment of African } \\
\text { American women to a home-based walking programme and } \\
\text { to examine the factors that contribute to attrition, eligibility, } \\
\text { and ineligibility during the recruitment screening protocol. }\end{array}$ & African American Women & 4 \\
\hline $\begin{array}{l}\text { 9. Baker et al, } \\
\text { 2008b }\end{array}$ & UK & $\mathrm{RCT}$ & $\begin{array}{l}\text { Effectiveness of pedometer based community walking } \\
\text { intervention on PA and health }\end{array}$ & $\begin{array}{l}\text { Community members in areas } \\
\text { of high deprivation (> 15\% } \\
\text { SIMD) }\end{array}$ & 3 \\
\hline $\begin{array}{l}\text { 10. Brownson et } \\
\text { al } 2005\end{array}$ & USA & $\mathrm{NRCT}$ & $\begin{array}{l}\text { To evaluate the impact of community based walking } \\
\text { approaches }\end{array}$ & Rural community members & 3 \\
\hline $\begin{array}{l}\text { 11. Cox et al, } \\
2008\end{array}$ & Australia & $\mathrm{RCT}$ & $\begin{array}{l}\text { Examine the effects of exercise mode and a behavioural } \\
\text { intervention on short and long-term retention and adherence. }\end{array}$ & $\begin{array}{l}\text { Previously sedentary older } \\
\text { women }\end{array}$ & 3 \\
\hline $\begin{array}{l}\text { 12. Dinger et al, } \\
2007\end{array}$ & USA & $\mathrm{RCT}$ & $\begin{array}{l}\text { Compare the effectiveness of two email delivered, pedometer } \\
\text { based interventions designed to increase walking and TTM } \\
\text { constructs among insufficiently active women. }\end{array}$ & $\begin{array}{l}\text { Insufficiently active women } \\
\text { (University staff and local } \\
\text { community members) }\end{array}$ & 3 \\
\hline $\begin{array}{l}\text { 13. Dubbert et } \\
\text { al, } 2002\end{array}$ & USA & $\mathrm{RCT}$ & $\begin{array}{l}\text { Effect of nurse counselling on walking for exercise in elderly } \\
\text { patients ( } 10 \text { months study) }\end{array}$ & Elderly primary care patients & 3 \\
\hline $\begin{array}{l}\text { 14. Dubbert et } \\
\text { al, } 2008\end{array}$ & USA & $\mathrm{RCT}$ & $\begin{array}{l}\text { To evaluate the effects of counselling linked with PHC visits on } \\
\text { walking and strength exercise in aging veterans }\end{array}$ & Elderly veterans & 3 \\
\hline $\begin{array}{l}\text { 15. Gilson et al, } \\
2008\end{array}$ & UK & $\begin{array}{l}\text { RCT, } \\
\text { Qualitative }\end{array}$ & $\begin{array}{l}\text { To compare two walking interventions and measure their } \\
\text { effect on daily step counts in a work-place environment }\end{array}$ & Work-place employees & 3 \\
\hline $\begin{array}{l}\text { 16. Jancey et al, } \\
2008\end{array}$ & Australia & $\begin{array}{l}\text { Before- } \\
\text { and-after } \\
\text { study }\end{array}$ & $\begin{array}{l}\text { To mobilise older adults into a neighbourhood-based walking } \\
\text { programme }\end{array}$ & Older adults & 3 \\
\hline $\begin{array}{l}\text { 17. Lamb et al, } \\
2002\end{array}$ & UK & $\mathrm{RCT}$ & To compare lead walks vs. advice only on PA (walking) & Middle aged adults & 3 \\
\hline $\begin{array}{l}\text { 18. Lee et al, } \\
1997\end{array}$ & USA & $\begin{array}{l}\text { RCT } \\
\text { Methods } \\
\text { paper }\end{array}$ & $\begin{array}{l}\text { To compare the efficacy of a mail versus phone based } \\
\text { behavioural intervention to promote walking for US adults }\end{array}$ & $\begin{array}{l}\text { Sedentary ethnic minority } \\
\text { women }\end{array}$ & 3 \\
\hline $\begin{array}{l}\text { 19. Matthews et } \\
\mathrm{al}, 2007\end{array}$ & USA & $\mathrm{RCT}$ & $\begin{array}{l}\text { To evaluate the effects of a 12-week home-based walking } \\
\text { intervention among breast cancer survivors }\end{array}$ & Breast cancer survivors & 3 \\
\hline $\begin{array}{l}\text { 20. Merom et al, } \\
2007\end{array}$ & Australia & $\mathrm{RCT}$ & $\begin{array}{l}\text { Efficacy of pedometers to act as a motivational tool in place of } \\
\text { face to face contact as part of a self-help package to increase } \\
\text { PA through walking. }\end{array}$ & Inactive adults & 3 \\
\hline $\begin{array}{l}\text { 21. Ornes and } \\
\text { Ransdell, } 2007\end{array}$ & USA & $\mathrm{RCT}$ & $\begin{array}{l}\text { To evaluate the impact of a web-based intervention for } \\
\text { women }\end{array}$ & Women & 3 \\
\hline
\end{tabular}




\section{Table 1 Characteristics of included studies (Continued)}

\begin{tabular}{|c|c|c|c|c|c|}
\hline $\begin{array}{l}\text { 22. Richardson et } \\
a l, 2007\end{array}$ & USA & RCT & $\begin{array}{l}\text { To compare the effects of structured and lifestyle goals in an } \\
\text { internet-mediated walking programme for adults with type } 2 \\
\text { diabetes }\end{array}$ & Adults with type 2 diabetes & 3 \\
\hline $\begin{array}{l}\text { 23. Rosenberg et } \\
\text { al, } 2009\end{array}$ & USA & $\begin{array}{l}\text { Before- } \\
\text { and-after } \\
\text { study }\end{array}$ & $\begin{array}{l}\text { Feasibility and acceptability of a novel multilevel walking } \\
\text { intervention for older adults in a continuing care retirement } \\
\text { community (CCRC). }\end{array}$ & Older adults & 3 \\
\hline $\begin{array}{l}\text { 24. Whitt-Glover } \\
\text { et al, } 2008\end{array}$ & USA & $\begin{array}{l}\text { Before- } \\
\text { and-after } \\
\text { study }\end{array}$ & $\begin{array}{l}\text { Feasibility and acceptability of implementing a physical activity } \\
\text { program for sedentary black adults in churches. (Information } \\
\text { sessions and lead walks) }\end{array}$ & Black adult, church attendees & 3 \\
\hline $\begin{array}{l}\text { 25. Arbour \& } \\
\text { Ginis, } 2009\end{array}$ & Canada & $\mathrm{RCT}$ & $\begin{array}{l}\text { Evaluate the effectiveness of implementation intentions on } \\
\text { walking behaviour }\end{array}$ & Women in the workplace & 2 \\
\hline $\begin{array}{l}\text { 26. Culos-Reed } \\
\text { et al, } 2008\end{array}$ & Canada & $\begin{array}{l}\text { Before- } \\
\text { and-after } \\
\text { study }\end{array}$ & $\begin{array}{l}\text { To assess the feasibility and health benefits of a mall walking } \\
\text { programme. }\end{array}$ & NS & 2 \\
\hline $\begin{array}{l}\text { 27. Currie and } \\
\text { Develin, } 2001\end{array}$ & Australia & $\begin{array}{l}\text { Before- } \\
\text { and-after } \\
\text { study }\end{array}$ & $\begin{array}{l}\text { To evaluate the impact of a community based pram walking } \\
\text { programme-organised pram walks. }\end{array}$ & Mothers and young children & 2 \\
\hline $\begin{array}{l}\text { 28. Darker et al, } \\
2010\end{array}$ & UK & $\mathrm{RCT}$ & $\begin{array}{l}\text { To examine whether altering perceived behavioural control } \\
\text { (PBC) affects walking ( } 6 / 7 \text { weeks). }\end{array}$ & NS & 2 \\
\hline $\begin{array}{l}\text { 29. De Cocker et } \\
\text { al } 2007\end{array}$ & Belgium & NRCT & Describe the effectiveness of the ' 10,000 steps Ghent' project. & $\begin{array}{l}\text { 'General population' adults in } \\
\text { a local community }\end{array}$ & 2 \\
\hline $\begin{array}{l}\text { 30. Dinger et al, } \\
2005\end{array}$ & USA & NRCT & $\begin{array}{l}\text { Examine the impact of a } 6 \text { week minimal contact intervention } \\
\text { on walking behaviour, TTM and self efficacy among women. }\end{array}$ & $\begin{array}{l}\text { Female employees or spouses } \\
\text { of university employees }\end{array}$ & 2 \\
\hline $\begin{array}{l}\text { 31. Engel and } \\
\text { Lindner, } 2006\end{array}$ & Australia & $\mathrm{RCT}$ & $\begin{array}{l}\text { To evaluate the effect of a pedometer intervention on adults } \\
\text { with type } 2 \text { diabetes }\end{array}$ & Adults with type 2 diabetes & 2 \\
\hline $\begin{array}{l}\text { 32. Foreman et } \\
\mathrm{al}, 2001\end{array}$ & Australia & Qualitative & $\begin{array}{l}\text { To increase the community's participation in physical activity } \\
\text { through group walking }\end{array}$ & Community members & 2 \\
\hline $\begin{array}{l}\text { 33. Humpel et al, } \\
2004\end{array}$ & Australia & $\mathrm{RCT}$ & $\begin{array}{l}\text { Examine the effectiveness of self-help print materials and } \\
\text { phone counselling in a study aimed specifically at promoting } \\
\text { walking for specific purposes }\end{array}$ & $\begin{array}{l}\text { Over } 40 \text { year old community } \\
\text { members }\end{array}$ & 2 \\
\hline $\begin{array}{l}\text { 34. Nies et al, } \\
2006\end{array}$ & USA & $\mathrm{RCT}$ & $\begin{array}{l}\text { To increase walking activity in sedentary women (Video } \\
\text { education, brief telephone calls without counselling, brief } \\
\text { telephone calls with counselling) }\end{array}$ & $\begin{array}{l}\text { European American and } \\
\text { African America women. }\end{array}$ & 2 \\
\hline $\begin{array}{l}\text { 35. Purath et al, } \\
2004\end{array}$ & USA & $\mathrm{RCT}$ & $\begin{array}{l}\text { To determine if a brief, tailored counselling intervention is } \\
\text { effective for increasing physical activity in sedentary women, in } \\
\text { the workplace }\end{array}$ & Women in the workplace & 2 \\
\hline $\begin{array}{l}\text { 36. Shaw et al, } \\
2007\end{array}$ & Australia & $\begin{array}{l}\text { Before- } \\
\text { and-after } \\
\text { study }\end{array}$ & To evaluate a workplace pedometer intervention & $\begin{array}{l}\text { Men and women in the } \\
\text { workplace }\end{array}$ & 2 \\
\hline $\begin{array}{l}\text { 37. Sidman et al, } \\
2004\end{array}$ & USA & $\begin{array}{l}\text { Before- } \\
\text { and-after } \\
\text { study }\end{array}$ & Promote physical activity through walking & Sedentary women & 2 \\
\hline $\begin{array}{l}\text { 38. Thomas and } \\
\text { Williams, } 2006\end{array}$ & Australia & $\begin{array}{l}\text { Before- } \\
\text { and-after } \\
\text { study }\end{array}$ & $\begin{array}{l}\text { Increase activity through wearing a pedometer and } \\
\text { encouraging participants to aim for } 10,000 \text { steps per day. }\end{array}$ & $\begin{array}{l}\text { Workplace staff (Excluding } \\
\text { hospital and community } \\
\text { services staff) }\end{array}$ & 2 \\
\hline $\begin{array}{l}\text { 39. Tudor-Locke } \\
\text { et al, } 2002\end{array}$ & USA & $\begin{array}{l}\text { Before- } \\
\text { and-after } \\
\text { study }\end{array}$ & Feasibility study of a community walking intervention & Sedentary diabetes sufferers & 2 \\
\hline $\begin{array}{l}\text { 40. Baker et al, } \\
\text { 2008a }\end{array}$ & UK & $\mathrm{RCT}$ & Examine the effectiveness of pedometers to motivate walking. & NS & 1 \\
\hline $\begin{array}{l}\text { 41. Hultquist et } \\
\text { al, } 2005\end{array}$ & USA & $\mathrm{RCT}$ & To compare the impact of two walking promotion messages & NS & 1 \\
\hline $\begin{array}{l}\text { 42. Lomabrd et } \\
\text { al, } 1995\end{array}$ & USA & $\mathrm{RCT}$ & To evaluate the effects of low $v$ high prompting for walking & NS & 1 \\
\hline $\begin{array}{l}\text { 43. DNSWH, } \\
2002\end{array}$ & Australia & $\begin{array}{l}\text { Before- } \\
\text { and-after } \\
\text { study }\end{array}$ & $\begin{array}{l}\text { To evaluate the impact of park modification, promotion of park } \\
\text { use and establishment of walking groups on physical activity } \\
\text { (including walking) }\end{array}$ & NS & 1 \\
\hline 44. Rovniak, 2005 & USA & $\begin{array}{l}\text { Before- } \\
\text { and-after } \\
\text { study }\end{array}$ & $\begin{array}{l}\text { Examine the extent to which theoretical fidelity influenced the } \\
\text { effectiveness of two walking programmes based on SCT. }\end{array}$ & NS & 1 \\
\hline
\end{tabular}


Table 1 Characteristics of included studies (Continued)

\begin{tabular}{clll}
\hline $\begin{array}{l}\text { 45. Rowley et al, UK } \\
2007\end{array}$ & $\begin{array}{l}\text { Before- } \\
\text { and-after } \\
\text { study } \\
\text { RCT }\end{array}$ & $\begin{array}{l}\text { To examine the development of two walking programmes by } \\
\text { a health visiting team to encourage undertaking of more } \\
\text { exercise. }\end{array}$ & $\begin{array}{l}\text { To evaluate the effects of a home based walking programme } \\
\text { with arthritis self-management education }\end{array}$ \\
$\begin{array}{c}\text { 47. Wyatt et al, USA } \\
2004\end{array}$ & $\begin{array}{l}\text { Before- } \\
\text { and-after } \\
\text { study }\end{array}$ & $\begin{array}{l}\text { Increasing lifestyle physical activity (i.e. walking) for weight gain } \\
\text { prevention }\end{array}$ & $\begin{array}{l}\text { Older adults } \\
\text { community }\end{array}$ \\
\hline
\end{tabular}

Forty studies reported a target population [27-45,48,50-60,62-65,68-70,72,73].

\section{Characteristics of the participants}

Thirty seven studies reported participant ages [28-30,32-47,49,51-54,56,58,59,62-67,70-73] with a mean age of 50.6 years, $(\mathrm{SD} \pm 8.1$ years), and a range of 18 to 92 years (Table 3 -Characteristics of participants). Sixteen out of forty two studies that reported gender data focused on recruiting female only participants $[27,29,30,32,35-37,41-44,46,51,52,55,68,70]$, with one study recruiting men only [34]. From the remaining twenty five studies that did not recruit sex specific groups, $70 \%$ (SD \pm 20.8 ) of participants were female. Twenty two studies reported data on nationality and ethnicity, of which seventeen reported descriptive statistics for ethnicity or race [27-38,40-42,46,49,51, $54,58,68,70,71]$. Three studies reported targeting one specific ethnic group, African-Americans [27,30,40]. Of the remaining studies, twelve reported other ethnicity data; $87 \%$ of these participants were white Caucasian [28,31-34,36,38,41,43,49,70,71]. Additional socio-demographic data (SES or income groups, education, urban/ rural living and relationship status) were reported but not consistently across all studies. Seven studies reported data on participant's income level data, which tended to be higher than average $[28,30,31,38,42,49,68]$. Sample sizes of the studies ranged from 9 to 1674 participants.

\section{Recruitment data reported}

Two studies reported all data for all components of recruitment, i.e. where recruitment took place; who conducted the recruitment; the time taken to conduct the planning/preparing and delivery stages [27,51]. Thirty nine studies did report a specified target group (Table 4-Recruitment planning/preparing and implementation). Forty four studies provide some details of where recruitment was conducted [27-49,51-56,58-67,69-73] but the recruitment location was often given vague descriptions, for example "in the community". Most popular were medical/care settings $(\mathrm{n}=12)$ [29-31,33,34,36, $38,43,49,51,55,63]$ or universities $(n=9)[37,40,41,43,44$, $46,47,62,70]$. Other community settings included for example, places of worship [67], hair salons [29], food establishments $[29,71]$ or specific events within such settings, for example meetings for new mothers [51].

Twenty one studies reported who conducted the study recruitment. Most popular recruiters were research staff $[28,31,33,34,37,39,51,52,54,62,64,67,72]$, often with assistance from health professionals like doctors or nurses $[29,33,51,65]$. Five studies reported using a dedicated "recruitment specialist" [27,30,35,51,69]. Only three studies reported the time spent planning/preparing their recruitment phases $[34,51,69]$. Eleven studies reported the time spent on implementing recruitment [27-30,32,51,62,63,71,72] and this averaged as 35 weeks, with a range of 2 days to 56 weeks.

\section{Recruitment procedures and approaches}

The reporting of recruitment methods was often sparse and unstructured (Table 5-Number of methods and types of recruitment procedures). Forty five studies provided data on the number of recruitment methods used (mean 2.7 , SD 1.97). Sixteen studies relied on one method of recruitment only $[33,34,43-45,50-53,56,58,60,62$, $64,65,72]$, and 26 studies used between two and four methods [27-32,35-41,54,55,63,66,69-71,73]. We identified two types of recruitment approaches, (i) active approaches; a recruitment method that requires those conducting the study to make the first contact with a participant (e.g. phone calls, face to face invitation, word of mouth, referrals), (ii) passive approaches; a recruitment method that requires a potential participant makes the first contact with the study (e.g. posters, leaflets drops, newspaper advertisements, mail outs). We did not observe any relationship between the quality of recruitment reporting and the number of recruitment strategies used. We did however observe that a number of studies used only passive techniques $(\mathrm{n}=21)[32,34,38,41,42,44$, $46-48,52,54,56,58-62,64,66,67,70]$, some used a mixture of active and passive techniques $(\mathrm{n}=22)$ [27-31,33,35-37, $39,40,49,53,55,57,63,65,68,69,71-73]$ and a small number used solely active only methods $(n=4)[43,45,50,51]$.

Passive recruitment methods, which require no interaction with the potential participants, were popular (Figure 2). Flyers/posters/advertisements/mail drops were the most cited approach used, appearing in 31 studies. 
Table 2 Assessment of study quality

\begin{tabular}{|c|c|c|c|c|c|c|}
\hline $\begin{array}{l}\text { Study } \\
\text { Author } \\
\text { (Year) }\end{array}$ & $\begin{array}{l}\text { Did the study report } \\
\text { where the } \\
\text { population was } \\
\text { recruited? }\end{array}$ & $\begin{array}{l}\text { Did the study } \\
\text { report who } \\
\text { conducted the } \\
\text { recruitment? }\end{array}$ & $\begin{array}{l}\text { Did the study report the } \\
\text { time spent planning/ } \\
\text { preparing the recruitment? }\end{array}$ & $\begin{array}{l}\text { Did the study report } \\
\text { the time spent } \\
\text { conducting the } \\
\text { recruitment? }\end{array}$ & $\begin{array}{l}\text { Did the study } \\
\text { target a specific } \\
\text { population? }\end{array}$ & $\begin{array}{l}\text { Quality } \\
\text { Metric } \\
\text { score }\end{array}$ \\
\hline $\begin{array}{l}\text { I. Watson } \\
\text { et al, } 2005\end{array}$ & Yes & Yes & Yes & Yes & Yes & 5 \\
\hline $\begin{array}{l}\text { 2. Banks- } \\
\text { Wallace } \\
\text { et al, } 2004\end{array}$ & Yes & Yes & No & Yes & Yes & 4 \\
\hline $\begin{array}{l}\text { 3. Kolt } \\
\text { et al, } 2006\end{array}$ & Yes & Yes & No & Yes & Yes & 4 \\
\hline $\begin{array}{l}\text { 4. Nguyen } \\
\text { et al, } 2002\end{array}$ & Yes & Yes & Yes & No & Yes & 4 \\
\hline $\begin{array}{l}5 . \\
\text { Prestwich } \\
\text { et al, } 2010\end{array}$ & Yes & Yes & No & Yes & Yes & 4 \\
\hline $\begin{array}{l}\text { 6. Rowland } \\
\text { et al, } 2004\end{array}$ & Yes & Yes & No & Yes & Yes & 4 \\
\hline $\begin{array}{l}\text { 7. Sherman } \\
\text { et al, } 2006\end{array}$ & Yes & Yes & No & Yes & Yes & 4 \\
\hline $\begin{array}{l}\text { 8. Wilbur } \\
\text { et al, } 2006\end{array}$ & Yes & Yes & No & Yes & Yes & 4 \\
\hline $\begin{array}{l}\text { 9. Baker } \\
\text { et al, 2008b }\end{array}$ & Yes & No & No & Yes & Yes & 3 \\
\hline $\begin{array}{l}10 . \\
\text { Brownson } \\
\text { et al } 2005\end{array}$ & Yes & Yes & No & No & Yes & 3 \\
\hline $\begin{array}{l}\text { 11. Cox } \\
\text { et al, } 2008\end{array}$ & Yes & Yes & No & No & Yes & 3 \\
\hline $\begin{array}{l}\text { 12. Dinger } \\
\text { et al, } 2007\end{array}$ & Yes & No & No & Yes & Yes & 3 \\
\hline $\begin{array}{l}13 . \\
\text { Dubbert } \\
\text { et al, } 2002\end{array}$ & Yes & Yes & No & No & Yes & 3 \\
\hline $\begin{array}{l}14 . \\
\text { Dubbert } \\
\text { et al, } 2008\end{array}$ & Yes & No & Yes & No & Yes & 3 \\
\hline $\begin{array}{l}\text { 15. Gilson } \\
\text { et al, } 2008\end{array}$ & Yes & Yes & No & No & Yes & 3 \\
\hline $\begin{array}{l}\text { 16. Jancey } \\
\text { et al, } 2008\end{array}$ & Yes & Yes & No & No & Yes & 3 \\
\hline $\begin{array}{l}\text { 17. Lamb } \\
\text { et al, } 2002\end{array}$ & Yes & Yes & No & No & Yes & 3 \\
\hline $\begin{array}{l}\text { 18. Lee } \\
\text { et al, } 1997\end{array}$ & Yes & Yes & No & $\mathrm{No}$ & Yes & 3 \\
\hline $\begin{array}{l}19 . \\
\text { Matthews } \\
\text { et al, } 2007\end{array}$ & Yes & Yes & No & No & Yes & 3 \\
\hline $\begin{array}{l}\text { 20. Merom } \\
\text { et al, } 2007\end{array}$ & Yes & Yes & No & No & Yes & 3 \\
\hline $\begin{array}{l}\text { 21. Ornes } \\
\text { and } \\
\text { Ransdell, } \\
2007\end{array}$ & Yes & Yes & No & No & Yes & 3 \\
\hline $\begin{array}{l}22 . \\
\text { Richardson } \\
\text { et al, } 2007\end{array}$ & Yes & Yes & No & No & Yes & 3 \\
\hline 23. & Yes & Yes & No & No & Yes & 3 \\
\hline
\end{tabular}


Table 2 Assessment of study quality (Continued)

\begin{tabular}{|c|c|c|c|c|c|c|}
\hline $\begin{array}{l}\text { 24. Whitt- } \\
\text { Glover } \\
\text { et al, } 2008\end{array}$ & Yes & Yes & No & $\mathrm{No}$ & Yes & 3 \\
\hline $\begin{array}{l}\text { 25. Arbour } \\
\text { \& Ginis, } \\
2009\end{array}$ & Yes & No & No & No & Yes & 2 \\
\hline $\begin{array}{l}\text { 26. Culos- } \\
\text { Reed et al, } \\
2008\end{array}$ & Yes & No & No & Yes & No & 2 \\
\hline $\begin{array}{l}\text { 27. Currie } \\
\text { and } \\
\text { Develin, } \\
2001\end{array}$ & Yes & No & No & No & Yes & 2 \\
\hline $\begin{array}{l}\text { 28. Darker } \\
\text { et al, } 2010\end{array}$ & Yes & No & No & Yes & No & 2 \\
\hline $\begin{array}{l}\text { 29. De } \\
\text { Cocker } \\
\text { et al } 2007\end{array}$ & Yes & No & No & No & Yes & 2 \\
\hline $\begin{array}{l}\text { 30. Dinger } \\
\text { et al, } 2005\end{array}$ & Yes & No & No & No & Yes & 2 \\
\hline $\begin{array}{l}\text { 31. Engel } \\
\text { and } \\
\text { Lindner, } \\
2006\end{array}$ & Yes & No & No & No & Yes & 2 \\
\hline $\begin{array}{l}\text { 32. Humpel } \\
\text { et al, } 2004\end{array}$ & Yes & No & No & No & Yes & 2 \\
\hline $\begin{array}{l}\text { 33. Nies } \\
\text { et al, } 2006\end{array}$ & Yes & No & No & No & Yes & 2 \\
\hline $\begin{array}{l}\text { 34. Purath } \\
\text { et al, } 2004\end{array}$ & Yes & No & No & No & Yes & 2 \\
\hline $\begin{array}{l}\text { 35. Shaw } \\
\text { et al, } 2007\end{array}$ & Yes & No & No & No & Yes & 2 \\
\hline $\begin{array}{l}\text { 36. Sidman } \\
\text { et al, } 2004\end{array}$ & Yes & No & No & No & Yes & 2 \\
\hline $\begin{array}{l}\text { 37. Thomas } \\
\text { and } \\
\text { Williams, } \\
2006\end{array}$ & Yes & No & No & No & Yes & 2 \\
\hline $\begin{array}{l}\text { 38. Tudor- } \\
\text { Locke et al, } \\
2002\end{array}$ & Yes & No & No & No & Yes & 2 \\
\hline $\begin{array}{l}39 . \\
\text { Foreman } \\
\text { et al, } 2001\end{array}$ & No & Yes & No & No & Yes & 2 \\
\hline $\begin{array}{l}\text { 40. Baker } \\
\text { et al, 2008a }\end{array}$ & Yes & No & No & No & No & 1 \\
\hline $\begin{array}{l}41 . \\
\text { Hultquist } \\
\text { et al, } 2005\end{array}$ & Yes & No & No & No & No & 1 \\
\hline $\begin{array}{l}42 . \\
\text { Lomabrd } \\
\text { et al, } 1995\end{array}$ & Yes & No & No & No & No & 1 \\
\hline $\begin{array}{l}43 . \\
\text { DNSWH, } \\
2002\end{array}$ & Yes & No & No & No & No & 1 \\
\hline $\begin{array}{l}\text { 44. Rovniak, } \\
2005\end{array}$ & Yes & No & No & No & No & 1 \\
\hline $\begin{array}{l}\text { 45. Rowley } \\
\text { et al, } 2007\end{array}$ & No & No & No & No & Yes & 1 \\
\hline $\begin{array}{l}\text { 46. Talbot } \\
\text { et al, } 2003\end{array}$ & Yes & No & No & No & No & 1 \\
\hline
\end{tabular}


Table 2 Assessment of study quality (Continued)

\begin{tabular}{|c|c|c|c|c|c|c|}
\hline $\begin{array}{l}\text { 47. Wyatt } \\
\text { et al, } 2004\end{array}$ & No & No & No & No & Yes & 1 \\
\hline Totals & 45 Yes 2 No & 22 Yes $25 \mathrm{No}$ & 3 Yes 44 No & 11 Yes 36 No & 40 Yes 7 No & \\
\hline
\end{tabular}

This was almost twice as prevalent as the second most popular approach, newsletters/newspaper articles $(\mathrm{n}=$ 18) and was nearly three times more frequently used than word of mouth. Word of mouth appeared in 12 studies, but we were unable to identify whether this was a proactive recruitment strategy or a reactive strategy, responding to low recruitment numbers. Less popular methods included medical and health insurance referral, invitations derived from clinical or employment data, study information sessions, resident listings, announcements at group meetings or community events and information stands.

\section{Locations for recruitment, interventions and target populations}

Table 6 presents data on the setting and location of recruitment and the study. We observed some studies that "matched" where the recruitment was conducted with where the intervention was delivered. Culos-Reed et al, 2008 reported recruiting participants for a mall walking study at the mall where the intervention was going to be delivered [71]. Other studies did not match in this way, and recruited in many different locations, often relying on print material alone, and requiring potential participants to attend a location which may not be easily accessible to them. Studies reported that they were "community-based" $(\mathrm{n}=25)$ [27-31,35,36,42,48-52,54-58,61,63,68,69,71-73] but asked community members to travel into a research setting to begin the process of participation; for example medical centres or universities $(\mathrm{n}=20)$ [29,30,33-38,41,43,46,47,49,56,62-67]. These interventions used a mixture of recruitment approaches including media events and led walking groups, face to face interventions (e.g. counselling, pedometers) or mediated interventions, such as internet, e-health and mobile phone technology [74].

\section{Recruitment rates and efficiencies}

We originally planned to calculate recruitment rates and efficiency ratios for each study but we were unable to do so due to missing data (Table 7 -Recruitment rates and efficiency ratios). Only three studies provided all the data points $[33,36,65]$. We were able to calculate a weekly recruitment rate using the final numbers of participants divided by the time spent recruiting in weeks for eleven studies (mean 38 participants per week, range 1 to 268 participants per week). We were not able to see any pattern between recruitment approaches and weekly rates. Two studies reported some data on the efforts needed to undertake recruitment. Jancey et al (2008) reported that after potential participants had received invitation cards it took approximately 9 calls to recruit one participant [53].

\section{Developing Recruitment Approaches}

We identified factors that may have helped or hindered recruitment from qualitative $[57,64,69]$ and protocol $[27,28,30,35]$ papers. These factors emerged as possible principles of recruitment and were related to training, engaging possible participants in the recruitment process and allowing sufficient time to pilot-test approaches. Watson et al (2009) used trained post-natal health care staff to actively recruit participants during their first home and health centres visits, and at group meetings for new mothers [51]. Recruitment approaches used by Banks-Wallace et al (2004) were based on a 5 month needs assessment study of the concerns and priorities of their target group [27]. The authors reported this process established trust between the research team and participants and ensured active participation in the study and in fact over-recruited from this population. Nguyen et al (2002) reported promoting participation via word of mouth, e.g. one participant tells/recruits another participant [69]. These appeared to have more impact on recruitment than passive approaches like posters or media stories [69]. These data suggest that developing recruitment approaches is a time and resource intensive activity, requiring skilled research and recruitment staff.

\section{Discussion}

We conducted a systematic review to examine the reported recruitment procedures of walking studies, in order to identify the characteristics of effective recruitment and the impact and differential effects of recruitment strategies among particular population groups. We identified the need for a common understanding of the recruitment process for walking studies in terms of conceptual definition, defining effectiveness and more detailed reporting. Due to the heterogeneity of studies we were not able to identify what specific recruitment approaches were most successful with particular population groups.

We identified eighteen recruitment strategies from 47 studies but did not see any relationship between one 
Table 3 Characteristics of participants

\begin{tabular}{|c|c|c|c|c|c|c|}
\hline $\begin{array}{l}\text { Study } \\
\text { Number, } \\
\text { Author and } \\
\text { Pub. Year }\end{array}$ & $\begin{array}{l}\text { Mean age, } \\
\text { SD or Range }\end{array}$ & $\begin{array}{l}\text { Gender }(\% \\
\text { Female) }\end{array}$ & Ethnicity & SES/Income & Education & $\begin{array}{l}\text { Quality } \\
\text { Metric } \\
\text { Score }\end{array}$ \\
\hline $\begin{array}{l}\text { I. Watson et } \\
\text { al, } 2005\end{array}$ & 29.4 & 100 & $\begin{array}{l}\text { NS ( } 20 \% \text { Not } \\
\text { Australian born) }\end{array}$ & $\begin{array}{l}96 \% \text { married, } 80 \% \text { Australian born. Competent at } \\
\text { filling in a questionnaire in English }\end{array}$ & $\begin{array}{l}39.2 \% \text { third level } \\
\text { education }\end{array}$ & 5 \\
\hline $\begin{array}{l}\text { 2. Banks- } \\
\text { Wallace et al, } \\
2004\end{array}$ & $18+$ & 100 & African American & NS & NS & 4 \\
\hline $\begin{array}{l}\text { 3. Kolt et al, } \\
2006\end{array}$ & $74(S D 6)$ & 66 & NS & $\begin{array}{l}\text { Urban, Patients from three GP lists. Phone lines at } \\
\text { home. }\end{array}$ & NS & 4 \\
\hline $\begin{array}{l}\text { 4. Nguyen et } \\
\text { al, } 2002\end{array}$ & NS & NS & NS & NS & NS & 4 \\
\hline $\begin{array}{l}\text { 5. Prestwich } \\
\text { et al, } 2010\end{array}$ & 23.44 & 64 & NS & Students & Undergraduate & 4 \\
\hline $\begin{array}{l}\text { 6. Rowland } \\
\text { et al, } 2004\end{array}$ & 74 (SD 6.2) & 69 & $\begin{array}{l}\text { White (Non-Hispanic) } \\
89 \%\end{array}$ & Income > 35 K US 26\%, Married, 57.5\%. & $\begin{array}{l}\text { Edu. > High } \\
\text { school diploma } \\
45 \%\end{array}$ & 4 \\
\hline $\begin{array}{l}\text { 7. Sherman } \\
\text { et al, } 2006\end{array}$ & $\begin{array}{l}42.5 \text { (Range } \\
22-64)\end{array}$ & 100 & Caucasian & $\begin{array}{l}\text { Rural, } 42 \% \text { Medicare, } 43 \% \text { private insurance, } 15 \% \\
\text { self pay or unknown insurance details, mean BMI } \\
30.6 \text { ( } 78 \% \text { overweight or obese), } 90 \% \text { with one or } \\
\text { more risk factors for CV disease, }\end{array}$ & NS & 4 \\
\hline $\begin{array}{l}\text { 8. Wilbur et } \\
\text { al, } 2006\end{array}$ & $\begin{array}{l}48.6 \text { (Range } \\
40-65)\end{array}$ & 100 & African American & $\begin{array}{l}\text { Urban, } 60 \% \text { unmarried, } 88 \% \text { mothers ( } 2.1 \text { children } \\
\text { ave.), } 70 \% \text { full time employed, } 61 \% \text { earning }>\$ 30 \\
\text { K annually, } 57 \% \text { reporting no 'hardhsips'. }\end{array}$ & $\begin{array}{l}87 \% \text { some or full } \\
\text { third level } \\
\text { education }\end{array}$ & 4 \\
\hline $\begin{array}{l}\text { 9. Baker et al, } \\
\text { 2008b }\end{array}$ & 49 (SD 9) & 78 & NS & NS & NS & 3 \\
\hline $\begin{array}{l}\text { 10. Brownson } \\
\text { et al } 2005\end{array}$ & $18+$ & 79.7 & $95 \%$ white & $31.3 \% 35 \mathrm{~K}+\mathrm{pa}$ & $\begin{array}{l}45 \% \text { some or full } \\
\text { third level Edu. }\end{array}$ & 3 \\
\hline $\begin{array}{l}\text { 11. Cox et al, } \\
2008\end{array}$ & $\begin{array}{l}55 \text { (Range } 50- \\
70 \text { ) }\end{array}$ & 100 & NS & $\begin{array}{l}\text { Urban, English Speakers, married ( } 76 \%) \text {, employed } \\
(56.5 \%) \text {, children (2.83). Non-smokers. }\end{array}$ & $\begin{array}{l}\text { Educated (13 } \\
\text { years ave.) }\end{array}$ & 3 \\
\hline $\begin{array}{l}\text { 12. Dinger et } \\
\text { al, } 2007\end{array}$ & $\begin{array}{l}41.5 \text { years } \\
\text { (Range } 25-54 \\
\text { years) }\end{array}$ & 100 & $86 \%$ White & Urban, BMI > 30 (57\%), access to email & $\begin{array}{l}68 \% \text { 3rd Level } \\
\text { Edu. }\end{array}$ & 3 \\
\hline $\begin{array}{l}\text { 13. Dubbert } \\
\text { et al, } 2002\end{array}$ & $\begin{array}{l}68.7 \text { yrs }(60- \\
80 \text { range) }\end{array}$ & $\begin{array}{l}1(99 \% \\
\text { Male) }\end{array}$ & 28\% Non-white & $\begin{array}{l}56.4 \% \text { rural, } 79.6 \% \text { married/cohabiting, } 12.7 \% \\
\text { tobacco users, } 8.8 \% \text { in financial hardship, } 7.4 \text { hrs } \\
\text { per week employment, } 20 \% \text { used alcohol, } 3.8 \text { co- } \\
\text { morbid medical conditions. }\end{array}$ & $\begin{array}{l}51.9 \% \text { high school } \\
\text { or more }\end{array}$ & 3 \\
\hline $\begin{array}{l}\text { 14. Dubbert } \\
\text { et al, } 2008\end{array}$ & $\begin{array}{l}\text { Mean } 72 \\
\text { (Range } 60 \text { to } \\
85 \text { years) }\end{array}$ & $\begin{array}{l}0(100 \% \\
\text { Male) }\end{array}$ & $\begin{array}{l}\text { 14\% African- } \\
\text { American, } 86 \% \text { White }\end{array}$ & Urban & $\begin{array}{l}\text { Majority high } \\
\text { school Educated }\end{array}$ & 3 \\
\hline $\begin{array}{l}\text { 15. Gilson et } \\
\text { al, } 2008\end{array}$ & 41.4 (SD 10.4) & $91 \%$ & NS & All employees at a University & NS & 3 \\
\hline $\begin{array}{l}\text { 16. Jancey et } \\
\text { al, } 2008\end{array}$ & $69(65-74)$ & 67 & NS & $\begin{array}{l}67 \% \text { Australian born, Urban ('Metropolitan Perth'), } \\
66 \% \text { had a partner }\end{array}$ & NS & 3 \\
\hline $\begin{array}{l}\text { 17. Lamb et } \\
\text { al, } 2002\end{array}$ & $\begin{array}{l}50.8 \text { (Range } \\
40-70)\end{array}$ & 52 & NS & NS & NS & 3 \\
\hline $\begin{array}{l}\text { 18. Lee et al, } \\
1997\end{array}$ & $\begin{array}{l}36.5 \text { (Range } \\
23-54 \text { ) }\end{array}$ & 100 & $\begin{array}{l}\text { Latino, African- } \\
\text { American, Asian, } \\
\text { Pacific Islanders, } \\
\text { other (ns) }\end{array}$ & "Middle class, well educated, English speaking" & "Well educated" & 3 \\
\hline $\begin{array}{l}\text { 19. Matthews } \\
\text { et al, } 2007\end{array}$ & 53 & 100 & $\begin{array}{l}84 \% \text { White. } 16 \% \\
\text { African-American/ } \\
\text { Other }\end{array}$ & NS & NS & 3 \\
\hline $\begin{array}{l}\text { 20. Merom et } \\
\text { al, } 2007\end{array}$ & $\begin{array}{l}49.1 \text { (Range } \\
30-65 \text { ) }\end{array}$ & 85 & NS & $\begin{array}{l}\text { Rural and Urban, } 74 \% \text { married, } 92.9 \text { English } \\
\text { speakers (primarily), } 57.7 \text { employed, } 72.2 \% \text { BMI > } \\
25,90 \% \text { non-smokers, } 81 \% \text { self rated health good } \\
\text { or more. }\end{array}$ & $\begin{array}{l}45.5 \% \text { university } \\
\text { degree }\end{array}$ & 3 \\
\hline $\begin{array}{l}\text { 21. Ornes } \\
\text { and Ransdell, } \\
2007\end{array}$ & 20 (SD 2.6) & 100 & $\begin{array}{l}\text { "Mostly Caucasian } \\
\text { volunteers" }\end{array}$ & Students & Undergraduate & 3 \\
\hline
\end{tabular}


Table 3 Characteristics of participants (Continued)

\begin{tabular}{|c|c|c|c|c|c|c|}
\hline $\begin{array}{l}22 . \\
\text { Richardson et } \\
\text { al, } 2007\end{array}$ & 52 (SD 10.5) & 65 & $\begin{array}{l}76 \% \text { white, } 13 \% \\
\text { black, } 10 \% \text { other }\end{array}$ & $64 \%$ high income $>\$ 70,000$ & NS & 3 \\
\hline $\begin{array}{l}23 . \\
\text { Rosenberg et } \\
\text { al, } 2009\end{array}$ & $\begin{array}{l}83 \text { (Range } 74- \\
92)\end{array}$ & $50 \%$ & NS & NS & NS & 3 \\
\hline $\begin{array}{l}\text { 24. Whitt- } \\
\text { Glover et al, } \\
2008\end{array}$ & $\begin{array}{l}\text { 52 (Range 20- } \\
\text { 83) }\end{array}$ & 89 & Black Americans & $\begin{array}{l}\text { Urban, average BMI 34.7, married (49\%), } 85 \% \text { had } \\
\text { at least one chronic health condition. }\end{array}$ & $\begin{array}{l}96 \% \text { high school } \\
\text { education or } \\
\text { higher }\end{array}$ & 3 \\
\hline $\begin{array}{l}\text { 25. Arbour \& } \\
\text { Ginis, } 2009\end{array}$ & 48.7 (SD 9.61) & 100 & $90 \%$ White & 90\% Employed & $\begin{array}{l}86 \% \text { Some or full } \\
\text { 3rd Level edu. }\end{array}$ & 2 \\
\hline $\begin{array}{l}\text { 26. Culos- } \\
\text { Reed et al, } \\
2008\end{array}$ & $\begin{array}{l}66 \text { (Range 46- } \\
\text { 83) }\end{array}$ & 81 & 96\% White & $76 \%$ retired, $70 \%$ higher education, urban & NS & 2 \\
\hline $\begin{array}{l}\text { 27. Currie } \\
\text { and Develin, } \\
2001\end{array}$ & NS & 100 & NS & NS & NS & 2 \\
\hline $\begin{array}{l}\text { 28. Darker et } \\
\text { al, } 2010\end{array}$ & $\begin{array}{l}40.6 \text { (Range } \\
16-65)\end{array}$ & 71 & NS & NS & NS & 2 \\
\hline $\begin{array}{l}\text { 29. De } \\
\text { Cocker et al } \\
2007\end{array}$ & $\begin{array}{l}48.7 \text { (Range } \\
25-75)\end{array}$ & 52.8 & NS & $\begin{array}{l}\text { Urban, } 68.1 \% \text { employed, } 63.7 \% \text { reporting good or } \\
\text { better than good health }\end{array}$ & $\begin{array}{l}60 \% \text { with third } \\
\text { level degrees }\end{array}$ & 2 \\
\hline $\begin{array}{l}\text { 30. Dinger et } \\
\text { al, } 2005\end{array}$ & $\begin{array}{l}41.7 \text { (SD 6.8) } \\
\text { (Range 25-54) }\end{array}$ & 100 & $89 \%$ White & $\begin{array}{l}\text { Employees or spouses of university employees, } \\
\text { Overweight or obese }(77.7 \%) \text {, not FT students, not } \\
\text { pregnant }\end{array}$ & $\begin{array}{l}\text { University degree } \\
(69 \%)\end{array}$ & 2 \\
\hline $\begin{array}{l}\text { 31. Engel and } \\
\text { Lindner, } 2006\end{array}$ & 62 & 46 & NS & NS & NS & 2 \\
\hline $\begin{array}{l}\text { 32. Foreman } \\
\text { et al, } 2001\end{array}$ & NS & $\begin{array}{l}\text { Male and } \\
\text { Female }\end{array}$ & NS & NS & NS & 2 \\
\hline $\begin{array}{l}\text { 33. Humpel } \\
\text { et al, } 2004\end{array}$ & 60 (SD 11) & $57 \%$ & NS & NS & $\begin{array}{l}46.9 \%<12 \text { yrs } \\
\text { edu., } 32.1 \% \text { had a } \\
\text { trade edu., } 21 \% \\
\text { Uni. }\end{array}$ & 2 \\
\hline $\begin{array}{l}\text { 34. Nies et al, } \\
2006\end{array}$ & $\begin{array}{l}45 \text { (Range 35- } \\
60 \text { ) }\end{array}$ & 100 & $\begin{array}{l}\text { European-American } \\
\text { and African-American }\end{array}$ & $\begin{array}{l}41 \%>50 \mathrm{~K} \text { (US) household income, } 49 \% \text { married, } \\
33 \% \text { southern American }\end{array}$ & $\begin{array}{l}74 \% \text { college edu. } \\
\text { or higher }\end{array}$ & 2 \\
\hline $\begin{array}{l}\text { 35. Purath et } \\
\text { al, } 2004\end{array}$ & 43.9 & 100 & 81.5\% White & $\begin{array}{l}100 \% \text { employed at a university ( } 62 \% \text { in admin/ } \\
\text { professional), } 92 \% \text { non-smokers, BMl } 30.5,68 \% \\
\text { married }\end{array}$ & $\begin{array}{l}14.25 \text { years edu. } \\
\text { (mean) }\end{array}$ & 2 \\
\hline $\begin{array}{l}\text { 36. Shaw et } \\
\text { al, } 2007\end{array}$ & 40 & 99 & NS & Employed in an urban workplace & NS & 2 \\
\hline $\begin{array}{l}\text { 37. Sidman } \\
\text { et al, } 2004\end{array}$ & 43.2 & 100 & NS & NS & NS & 2 \\
\hline $\begin{array}{l}\text { 38. Thomas } \\
\text { and Williams, } \\
2006\end{array}$ & $18-50+$ & 75.5 & NS & $\begin{array}{l}\text { Employed, Both Urban and Rural locations. 'wide } \\
\text { variety of professions, ages, incomes, education } \\
\text { standards and levels of health and fitness not } \\
\text { considered, disadvantaged in terms of the social } \\
\text { determinants of health' 'almost all could be } \\
\text { described as sedentary' }\end{array}$ & NS & 2 \\
\hline $\begin{array}{l}\text { 39. Tudor- } \\
\text { Locke et al, } \\
2002\end{array}$ & 53 (SD 6) & 66 & NS & NS & NS & 2 \\
\hline $\begin{array}{l}\text { 40. Baker et } \\
\text { al, 2008a }\end{array}$ & 40 (SD 8.6) & 86 & NS & NS & NS & 1 \\
\hline $\begin{array}{l}\text { 41. Hultquist } \\
\text { et al, } 2005\end{array}$ & 45 (SD 6 yrs) & 100 & $\begin{array}{l}3 \text { non-white among } \\
\text { completers }\end{array}$ & NS & NS & 1 \\
\hline $\begin{array}{l}\text { 42. Lomabrd } \\
\text { et al, } 1995\end{array}$ & 40 (SD 9) & 98 & NS & University staff & NS & 1 \\
\hline $\begin{array}{l}\text { 43. DNSWH, } \\
2002\end{array}$ & (Range 25-65) & NS & NS & Suburban & NS & 1 \\
\hline
\end{tabular}


Table 3 Characteristics of participants (Continued)

\begin{tabular}{|c|c|c|c|c|c|c|}
\hline $\begin{array}{l}\text { 44. Rovniak, } \\
2005\end{array}$ & $\begin{array}{l}\text { Men (Range } \\
\text { 20-44) } \\
\text { Women } \\
\text { (Range 20-54) }\end{array}$ & 93.5 & NS & $\begin{array}{l}\text { Urban, at least access to email, sedentary, no } \\
\text { more than one health risk factor, BMI }<39.9 \text {, no } \\
\text { metabolic, pulmonary or CV disease, no bone } \\
\text { joint or foot problems, not pregnant. }\end{array}$ & NS & 1 \\
\hline $\begin{array}{l}\text { 45. Rowley et } \\
\text { al, } 2007\end{array}$ & $\begin{array}{l}\text { Children 0-4 } \\
\text { Adults not } \\
\text { reported }\end{array}$ & $\begin{array}{l}100 \\
\text { (Adults) } \\
\text { Children } \\
\text { not } \\
\text { reported }\end{array}$ & $\begin{array}{l}\text { There were no } \\
\text { children or babies } \\
\text { from ethnic minority } \\
\text { groups'. }\end{array}$ & Affluent' & NS & 1 \\
\hline $\begin{array}{l}\text { 46. Talbot et } \\
\text { al, } 2003\end{array}$ & 69 (SD 6) & 76 & 17\% Non-White & $60 \%>\$ 30 \mathrm{~K} \mathrm{pa}$ & NS & 1 \\
\hline $\begin{array}{l}\text { 47. Wyatt et } \\
\text { al, } 2004\end{array}$ & NS & NS & NS & NS & NS & 1 \\
\hline
\end{tabular}

particular strategy or group of strategies and recruitment rates. Many studies blended different recruitment approaches and strategies, adopting an almost "trial and error" approach. Only two studies reported the effectiveness of their approaches to recruitment [28,35]. We were able to distinguish active and passive recruitment approaches. Further research is needed to directly compare specific recruitment strategies.

Very few studies examined the successes of recruitment approaches to physical activity interventions. Harris et al (2008) conducted a randomized controlled trial of four recruitment strategies in their physical activity promotion intervention study for older adults [75]. The authors reported that telephone follow up a week post invitation significantly increased recruitment compared to invitation only. Certainly the principle of follow up was found in a number of our included studies $[53,63,72]$ but we could not assess the efficacy of these strategies. The efficacy of phone recruitment has been questioned by Margitic et al (1999) [76] who compared three recruitment strategies for Project ACT: patient mailings, office-based questionnaires and telephone contact. However their participants were not randomized to a particular strategy. The authors also reported that despite telephone recruitment appearing to be productive this strategy was dropped in two out of eight recruitment sites on cost grounds. This behavioural approach of using phone follow up has previously been reported to be more effective than no follow up in changing physical activity and walking behaviour $[10,23]$ and certainly warrants further testing in terms of a possible recruitment strategy.

Tai and Iliffe's (2000) experiences of conducting physical activity studies also support our observation that piloting and pre-testing of recruitment methods would improve rates of recruitment and precision in recruiting specific target groups [77]. Our review clearly shows that current recruitment strategies resulted in recruiting mostly white, well-educated, middle aged women. The attraction of walking projects to particular social groups has also been reported in previous evaluation studies of community walking programmes both in the UK [78] and USA $[27,31]$. Our review found that recruitment rates were poorer for men, especially within workplace or community settings but we were unable to determine if it is a fault of the recruitment, or the offer of walking or a combination of both that is at fault.

We identified a number of studies that "matched" where the recruitment was conducted, with where the intervention was delivered. This principle supports the notion that connecting the place of recruitment and intervention may be more efficient both for the participants, recruiters and interventions teams. We found studies that did not effectively match these aspects and perhaps this was reflected in the total number of participants recruited and the longer time it took to conduct their recruitment phase. For example, Baker et al (2008) reported that participants were expected to travel to the university to receive their intervention. We found little data on the time spent planning/preparing and implementing recruitment so any potential learning from recruitment remains unreported [63].

We identified a number of studies that also aimed to match those recruiting with those being recruited. Banks-Wallace et al (2004) reported in detail their use of a recruitment mediator [27]. The mediator was the same gender as the target group, was a prominent local figure, trained in delivering community interventions and female. Her role was to introduce the study to key significant figures in the area and increase awareness, to assist directly with the recruitment phase and to introduce the researchers to the potential participants at an information session. Banks-Wallace et al (2004) described this approach as increasing trust and decreasing differences between the recruiters and recruited [27].

Our review clearly found there was very little consistency in the definition or reporting of recruitment. We found many different interpretations of (i) what is the recruitment process? and (ii) what is an appropriate metric for evaluating the effectiveness of recruitment? 
Table 4 Recruitment planning and implementation (Quality Metric categories)

\begin{tabular}{|c|c|c|c|c|c|c|}
\hline $\begin{array}{l}\text { Study } \\
\text { Author } \\
\text { (Year) }\end{array}$ & $\begin{array}{l}\text { Where did the Recruitment } \\
\text { take place? }\end{array}$ & Who did the Recruitment? & $\begin{array}{l}\text { Time spent Planning/ } \\
\text { Preparing recruitment }\end{array}$ & $\begin{array}{l}\text { Time spent } \\
\text { Executing } \\
\text { recruitment } \\
\text { (Weeks) }\end{array}$ & $\begin{array}{l}\text { Population } \\
\text { Targeted } \\
\text { (Yes/No)? }\end{array}$ & $\begin{array}{l}\text { Quality } \\
\text { Metric } \\
\text { score }\end{array}$ \\
\hline $\begin{array}{l}\text { I. Watson } \\
\text { et al, } 2005\end{array}$ & $\begin{array}{l}\text { Home, health centre visits, at } \\
\text { mothers group meetings }\end{array}$ & $\begin{array}{l}\text { Nurses trained in recruitment } \\
\text { and research staff }\end{array}$ & $\begin{array}{l}1 \text { month including all } \\
\text { training of nurses and } \\
\text { intervention by } \\
\text { researchers to help with } \\
\text { recruitment difficulties. }\end{array}$ & 6 weeks & Yes & 5 \\
\hline $\begin{array}{l}\text { 2. Banks- } \\
\text { Wallace et } \\
\text { al, } 2004\end{array}$ & $\begin{array}{l}\text { In the community at venues } \\
\text { typically used for hosting African } \\
\text { American community events }\end{array}$ & Recruitment Protocol Specialist & NS & 21.6 weeks & Yes & 4 \\
\hline $\begin{array}{l}\text { 3. Kolt et } \\
\text { al, } 2006\end{array}$ & $\begin{array}{l}\text { By mail and a follow up home } \\
\text { visit }\end{array}$ & Researchers & NS & 39 weeks & Yes & 4 \\
\hline $\begin{array}{l}\text { 4. Nguyen } \\
\text { et al, } 2002\end{array}$ & $\begin{array}{l}\text { Mainly passively in the } \\
\text { community but also used press } \\
\text { conferences and info/taster } \\
\text { sessions }\end{array}$ & Public health official & $\begin{array}{l}3 \text { years (Rolling } \\
\text { development) }\end{array}$ & NS & Yes & 4 \\
\hline $\begin{array}{l}5 . \\
\text { Prestwich } \\
\text { et al, } 2010\end{array}$ & University & Researchers & NS & 2.5 weeks & Yes & 4 \\
\hline $\begin{array}{l}\text { 6. Rowland } \\
\text { et al, } 2004\end{array}$ & $\begin{array}{l}\text { Via telephone, direct mail and } \\
\text { then at multiple locations and } \\
\text { media in the community }\end{array}$ & Research team members & NS & 43.3 weeks & Yes & 4 \\
\hline $\begin{array}{l}\text { 7. Sherman } \\
\text { et al, } 2006\end{array}$ & $\begin{array}{l}\text { In a clinic, hair salons-and food } \\
\text { establishments }\end{array}$ & Nurses & NS & 0.28 weeks & Yes & 4 \\
\hline $\begin{array}{l}\text { 8. Wilbur et } \\
\text { al, } 2006\end{array}$ & $\begin{array}{l}\text { Two federally qualified } \\
\text { community health centres serving } \\
\text { poor and working class urban } \\
\text { populations. Screening and data } \\
\text { collection was carried out here to } \\
\text { reduce power differences } \\
\text { (perceived) and increase trust. } \\
\text { Concentrated on an area within a } \\
\text { 3-mile radius of the data } \\
\text { collection sites. Also interacted in } \\
\text { the community at health fairs } \\
\text { and presentations. }\end{array}$ & $\begin{array}{l}\text { Team specifically set up to } \\
\text { deliver the recruitment made up } \\
\text { of AA female nurses, either living } \\
\text { in the community or who had } \\
\text { family ties to the community. }\end{array}$ & NS & 121.3 weeks & Yes & 4 \\
\hline $\begin{array}{l}\text { 9. Baker et } \\
\text { al, 2008b }\end{array}$ & $\begin{array}{l}\text { Local community, GP surgeries, } \\
\text { shops, community stalls }\end{array}$ & NS & NS & 21.6 months & Yes & 3 \\
\hline $\begin{array}{l}10 . \\
\text { Brownson } \\
\text { et al } 2005\end{array}$ & $\begin{array}{l}\text { Through media, at physicians } \\
\text { practices, at community centres, } \\
\text { on walking routes, in the } \\
\text { community active and passively }\end{array}$ & $\begin{array}{l}\text { Community organisation staff, } \\
\text { research staff, physicians }\end{array}$ & NS & NS & Yes & 3 \\
\hline $\begin{array}{l}\text { 11. Cox et } \\
\text { al, } 2008\end{array}$ & $\begin{array}{l}\text { Ads delivered in the community. } \\
\text { Screening took place at the } \\
\text { community centre }\end{array}$ & Research assistants & NS & NS & Yes & 3 \\
\hline $\begin{array}{l}\text { 12. Dinger } \\
\text { et al, } 2007\end{array}$ & Local media and electronically & NS & NS & 4.3 weeks & Yes & 3 \\
\hline $\begin{array}{l}13 . \\
\text { Dubbert et } \\
\text { al, } 2002\end{array}$ & By mail, phone and at the clinic & Researchers and Research Nurse & NS & NS & Yes & 3 \\
\hline $\begin{array}{l}14 . \\
\text { Dubbert et } \\
\text { al, } 2008\end{array}$ & $\begin{array}{l}\text { Primary care medical centre as } \\
\text { part of routine care }\end{array}$ & NS & 2 to 3 years & NS & Yes & 3 \\
\hline $\begin{array}{l}\text { 15. Gilson } \\
\text { et al, } 2008\end{array}$ & Via work email & Researchers & NS & NS & Yes & 3 \\
\hline $\begin{array}{l}\text { 16. Jancey } \\
\text { et al, } 2008\end{array}$ & $\begin{array}{l}\text { Over the phone to home phone } \\
\text { numbers }\end{array}$ & Researchers & NS & NS & Yes & 3 \\
\hline $\begin{array}{l}\text { 17. Lamb } \\
\text { et al, } 2002\end{array}$ & $\begin{array}{l}\text { Via post, phone and info sessions } \\
\text { at primary care setting }\end{array}$ & $\begin{array}{l}\text { Researchers, via GP, and staff } \\
\text { nurse }\end{array}$ & NS & NS & Yes & 3 \\
\hline $\begin{array}{l}\text { 18. Lee et } \\
\text { al, } 1997\end{array}$ & $\begin{array}{l}\text { Directly and indirectly in the } \\
\text { community }\end{array}$ & $\begin{array}{l}\text { Female students trained in } \\
\text { recruitment methods }\end{array}$ & NS & NS & Yes & 3 \\
\hline
\end{tabular}


Table 4 Recruitment planning and implementation (Quality Metric categories) (Continued)

\begin{tabular}{|c|c|c|c|c|c|c|}
\hline $\begin{array}{l}19 . \\
\text { Matthews } \\
\text { et al, } 2007\end{array}$ & Clinic & Clinical staff & NS & NS & Yes & 3 \\
\hline $\begin{array}{l}\text { 20. Merom } \\
\text { et al, } 2007\end{array}$ & $\begin{array}{l}\text { Passively in the community and } \\
\text { actively by phone via another } \\
\text { study }\end{array}$ & $\begin{array}{l}\text { Researchers in the NSW Health } \\
\text { survey (recruitment by proxy) } \\
\text { and researchers on this study }\end{array}$ & NS & NS & Yes & 3 \\
\hline $\begin{array}{l}\text { 21. Ornes } \\
\text { and } \\
\text { Ransdell, } \\
2007\end{array}$ & University campus & Researchers & NS & NS & Yes & 3 \\
\hline $\begin{array}{l}22 . \\
\text { Richardson } \\
\text { et al, } 2007\end{array}$ & Medical centre & Physicians & NS & NS & Yes & 3 \\
\hline $\begin{array}{l}23 . \\
\text { Rosenberg } \\
\text { et al, } 2009\end{array}$ & Care community & Researchers & NS & NS & Yes & 3 \\
\hline $\begin{array}{l}\text { 24. Arbour } \\
\& \text { Ginis, } \\
2009\end{array}$ & University and Local Community & NS & NS & NS & Yes & 2 \\
\hline $\begin{array}{l}\text { 25. Baker et } \\
\text { al, 2008a }\end{array}$ & University campus & NS & NS & NS & NS & 2 \\
\hline $\begin{array}{l}\text { 26. Culos- } \\
\text { Reed et al, } \\
2008\end{array}$ & $\begin{array}{l}\text { In the community and at the } \\
\text { malls }\end{array}$ & NS & NS & 2 weeks & No & 2 \\
\hline $\begin{array}{l}\text { 27. Currie } \\
\text { and } \\
\text { Develin, } \\
2001\end{array}$ & $\begin{array}{l}\text { Places where pre and post natal } \\
\text { mums engage with health care, } \\
\text { shopping and school }\end{array}$ & NS & NS & NS & Yes & 2 \\
\hline $\begin{array}{l}\text { 28. Darker } \\
\text { et al, } 2010\end{array}$ & In the local media (Passive) & NS & NS & 30.3 weeks & No & 2 \\
\hline $\begin{array}{l}\text { 29. De } \\
\text { Cocker et } \\
\text { al } 2007\end{array}$ & $\begin{array}{l}\text { By mail or phone to participants } \\
\text { homes. Indirect but active }\end{array}$ & NS & NS & NS & Yes & 2 \\
\hline $\begin{array}{l}\text { 30. Dinger } \\
\text { et al, } 2005\end{array}$ & University & NS & NS & NS & Yes & 2 \\
\hline $\begin{array}{l}\text { 31. Engel } \\
\text { and } \\
\text { Lindner, } \\
2006\end{array}$ & In community via newspapers & NS & NS & NS & Yes & 2 \\
\hline $\begin{array}{l}32 . \\
\text { Foreman et } \\
\text { al, } 2001\end{array}$ & NS & Walk leaders and organisers & NS & NS & Yes & 2 \\
\hline $\begin{array}{l}\text { 33. Humpel } \\
\text { et al, } 2004\end{array}$ & Via post. No face to face & NS & NS & NS & Yes & 2 \\
\hline $\begin{array}{l}\text { 34. Nies et } \\
\text { al, } 2006\end{array}$ & $\begin{array}{l}\text { Through media and fliers in the } \\
\text { community }\end{array}$ & NS & NS & NS & Yes & 2 \\
\hline $\begin{array}{l}\text { 35. Purath } \\
\text { et al, } 2004\end{array}$ & $\begin{array}{l}\text { Health screening day within a } \\
\text { university }\end{array}$ & NS & NS & NS & Yes & 2 \\
\hline $\begin{array}{l}\text { 36. Shaw et } \\
\text { al, } 2007\end{array}$ & Workplace (Health centre) & NS & NS & NS & Yes & 2 \\
\hline $\begin{array}{l}\text { 37. Sidman } \\
\text { et al, } 2004\end{array}$ & Two University campuses & NS & NS & NS & Yes & 2 \\
\hline $\begin{array}{l}\text { 38. Thomas } \\
\text { and } \\
\text { Williams, } \\
2006\end{array}$ & Workplace (Electronically) & NS & NS & NS & Yes & 2 \\
\hline $\begin{array}{l}\text { 39. Tudor- } \\
\text { Locke et al, } \\
2002\end{array}$ & Diabetes Centre & NS & NS & NS & Yes & 2 \\
\hline 40. Whitt- & At churches & Church pastors and researchers & NS & NS & Yes & 2 \\
\hline
\end{tabular}
al, 2008 
Table 4 Recruitment planning and implementation (Quality Metric categories) (Continued)

\begin{tabular}{|c|c|c|c|c|c|c|}
\hline $\begin{array}{l}41 . \\
\text { Hultquist et } \\
\text { al, } 2005\end{array}$ & University & NS & NS & NS & No & 1 \\
\hline $\begin{array}{l}42 . \\
\text { Lomabrd } \\
\text { et al, } 1995\end{array}$ & University campus & NS & NS & NS & No & 1 \\
\hline $\begin{array}{l}43 . \\
\text { DNSWH, } \\
2002\end{array}$ & $\begin{array}{l}\text { In local area via media and } \\
\text { advertising and information }\end{array}$ & NS & NS & NS & No & 1 \\
\hline $\begin{array}{l}\text { 44. Rovniak, } \\
2005\end{array}$ & $\begin{array}{l}\text { At multiple locations in the } \\
\text { community. Mainly passive. }\end{array}$ & NS & NS & NS & No & 1 \\
\hline $\begin{array}{l}\text { 45. Rowley } \\
\text { et al, } 2007\end{array}$ & NS & NS & NS & NS & Yes & 1 \\
\hline $\begin{array}{l}\text { 46. Talbot } \\
\text { et al, } 2003\end{array}$ & $\begin{array}{l}\text { Senior centres, ads in local } \\
\text { newspapers }\end{array}$ & NS & NS & NS & NS & 1 \\
\hline $\begin{array}{l}\text { 47. Wyatt } \\
\text { et al, } 2004\end{array}$ & NS & NS & NS & NS & Yes & 1 \\
\hline
\end{tabular}

The lack of conceptual clarity about recruitment as a process is surprising and potentially impacts on costeffectiveness. The RE-AIM framework emphasises the need to judge the success of an intervention from both the reach and uptake of an intervention [79]. In light of this we constructed a conceptual framework for our review by defining the stages of recruitment and potential pool of participants (Figure 3). This framework offers a starting point for further debate and refinement. The framework offers a clear concept of the stages and steps of recruitment and the chance to record the numbers of participants at each stage and action.

Our framework divided recruitment into two phases, planning/preparing and implementation, with four stages involving discrete actions by researchers/recruiters, (i) identification of participant pools, (ii) invitation and monitor response and uptake, (iii) assessment, screening and facilitation and (iv) re-invitation of responders, before the delivery of intervention to starters. This framework highlights the actions needed at the start of a recruitment process, i.e. planning/preparing the recruitment process. It also emphasises the importance of the reach of an intervention i.e. the pool of participants used to provide recruits. This differs from the recent new CONSORT framework which asks for dates of recruitment period (i.e. delivery) and enrolment stage [18]. CONSORT stipulates data must be reported for numbers of participants eligible for study which we feel not only ignores the overall pool of possible participants, especially in community based studies of walking interventions, but also ignores the population deemed ineligible, as seen in pre-screenings of patient lists for existing conditions [18]. The "pool" of participants perhaps provides a more realistic denominator for assessing overall recruitment rates. This metric will allow new studies to (i) consider if the recruitment was efficient (i.e. study recruited expected numbers of participants) and/or (ii) consider if it was effective (i.e. study recruited the right target group), and/or (iii) reflect the true costs of all recruitment actions within overall cost benefit calculations. The need for better reporting of recruitment actions and numbers is essential to improve the assessment of present recruitment strategies. This view is mirrored in recruitment studies of other health behaviours, and better reporting must begin before we can start to identify which strategies provide the best recruitment rates [80].

The results of our review were limited to walking intervention studies. We were limited by only including studies written in the English language. We were limited by what was reported in papers but our consistent application of inclusion, quality and data extraction criteria have illustrated the need for improvement in both the reporting and science of recruitment. As journals look to keep research reports within word limits, it is likely that there will continue to be a lack of journal space to report recruitment details, and we would like to call on editors and authors to report recruitment details or provide short methods papers for the insight of future researchers. As far as we are aware this review is the first of its kind focusing on one domain of physical activity behaviour. The lack of understanding and studies into recruitment may reflect some of the findings about the existing weaknesses of the evidence base for walking interventions, e.g. lack of generalisability of interventions across different social groups [10].

The evidence base for the benefits of walking is now expanding but until it is clearer what strategies are effective in both recruiting and initiating people to begin walking, such benefits may be out of reach for particular population groups. Practitioners would benefit from the assurance of having an evidence based best 
Table 5 Recruitment planning and implementation (Quality Metric categories)

\begin{tabular}{|c|c|c|c|c|}
\hline $\begin{array}{l}\text { Study Author } \\
\text { (Year) }\end{array}$ & $\begin{array}{l}\text { No. Of } \\
\text { Methods } \\
\text { Used }\end{array}$ & $\begin{array}{l}\text { Procedures including who conducted the recruitment, where it took } \\
\text { place and what was done }\end{array}$ & $\begin{array}{l}\text { Active, passive or a } \\
\text { mixture of } \\
\text { approaches }\end{array}$ & $\begin{array}{l}\text { Quality } \\
\text { Metric } \\
\text { Score }\end{array}$ \\
\hline $\begin{array}{l}\text { I. Watson et al, } \\
2005\end{array}$ & 1 & $\begin{array}{l}\text { Nurse conducted face to face recruitment at clinics, mothers' group meetings } \\
\text { and home visits. }\end{array}$ & Active & 5 \\
\hline $\begin{array}{l}\text { 2. Banks- } \\
\text { Wallace et al, } \\
2004\end{array}$ & 4 & $\begin{array}{l}\text { Researchers placed flyers in church bulletins and the community, health } \\
\text { practitioner referrals were generated, word of mouth was used and } \\
\text { structured pre-intervention meetings took place. }\end{array}$ & Passive/Active & 4 \\
\hline $\begin{array}{l}\text { 3. Kolt et al, } \\
2006\end{array}$ & 1 & $\begin{array}{l}\text { A three phased and sequenced approach was conducted by the researchers, } \\
\text { the GP and staff nurse. An invitation letter was sent from the GP surgery a } \\
\text { pre-paid response card for those expressing interest. Follow up screening } \\
\text { calls then follow up visits to provide info and gain consent. }\end{array}$ & Passive/Active & 4 \\
\hline $\begin{array}{l}\text { 4. Nguyen et al, } \\
2002\end{array}$ & 3 & $\begin{array}{l}\text { A public health official co-ordinated the recruitment and used the local } \\
\text { media, network construction and face to face recruitment of volunteer walk } \\
\text { leaders. Press conferences and promotional materials were sent to local } \\
\text { media outlets, community health centres, libraries, senior's club networks to } \\
\text { promote the club. Leaflets on local community settings, ads in free } \\
\text { newspapers, promotional messages placed on light panels around the city, } \\
\text { community TV ads and features, press releases for local media, newsletters, } \\
\text { press conference, celebration events. Comments elsewhere stated that face } \\
\text { to face recruitment was the most successful for this study, but this was only } \\
\text { used to recruit walk leaders. }\end{array}$ & Passive/Active & 4 \\
\hline $\begin{array}{l}\text { 5. Prestwich et } \\
\text { al, } 2010\end{array}$ & 1 & $\begin{array}{l}\text { Researchers sent emails to the current students at their university. Course } \\
\text { credit or cash were used as an incentive. }\end{array}$ & Passive & 4 \\
\hline 6. Rowland et & 11 & $\begin{array}{l}\text { Computer assisted telephone interviews (CATI) was initially conducted by } \\
\text { researchers. A database of potential participants was screened for telephor }\end{array}$ & Active/Passive & 4 \\
\hline
\end{tabular}

7. Sherman et 2

al, 2006

8. Wilbur et al, 3 2006

\section{Baker et al, 4} $2008 \mathrm{~b}$

10. Brownson 8 et al 2005

11. Cox et al, 1 2008 researchers. A database of potential participants was screened for telephone numbers. If this was not successful in recruiting the sample size needed the direct mailing was used. Finally, to complete the sample size quota canvassing in the local community (including face to face, door to door, posters and flyers at churches and senior housing units, snowballing, utilising 'community brokers', and newspapers) was conducted. Recruitment was systematic, purposeful and carried out in the order described but was somewhat inequitable as the first screening criterion was the availability of a phone number. It also required significant community assistance to reach those harder to engage.

Active recruitment by a nurse at a health clinic, advertisements in hair salons and food establishments. The paper states that the 'main source of recruitment came from advertisements in the community and word of mouth'.

Researchers designed a flyer with community input and received advice on where to place it. Emails and newspaper announcements were also used. Recruitment staff distributed print material at specified schools, churches, grocery shops, libraries, clinics, community agencies and community fairs and at 10 presentations in community agencies, clinics, and churches. Email announcement at local medical centre workplaces and an announcement in the community newspaper were used. A good aim of matching the invitation to the invitee and finding the best place to distribute it was a positive here. Unfortunately word of mouth wasn't actively used or reported and only the research team recruitment staff acted as recruiters for face to face recruitment.

Mail drops were carried out and adverts were placed in local papers and P. up. This approach was modified and expanded throughout the recruitment phase as the researchers identified their lack of impact on the target group. However, the methods were mainly passive and not altered to be more engaging or mediating with the target group. It is not specifically stated who conducted the recruitment.

Recruitment was initially by proxy during a baseline survey for another piece Passive/Active also promoted at community events, by physician recommendation, trail signage advertising and word or mouth. Recruitment methods were not explicitly reported but intervention communities used participatory approaches to develop their intervention options. Taster events, one off walks, clean up trail days, and 5 media events were held.

Research assistants placed advertisements in the local community.

Passive 
Table 5 Recruitment planning and implementation (Quality Metric categories) (Continued)

12. Dinger et al, 3
2007
13. Dubbert et 1
al, 2002

al, 2002

\author{
14. Dubbert et 1 \\ al, 2008 \\ 15. Gilson et al, 1 \\ 2008 \\ 16. Jancey et al, 1 \\ 2008
}

17. Lamb et al, 1 2002

18. Lee et al, 4 1997

19. Matthews et 3 al, 2007

20. Merom et
al, 2007
21. Ornes and
Ransdell, 2007
22. Richardson
et al, 2007

23. Rosenberg 2 et al, 2009

24. Whitt-Glover 5 et al, 2008

25. Arbour \& 2
Ginis, 2009
26. Culos-Reed 4
et al, 2008
27. Currie and 4

Develin, 2001

28. Darker et al, 2 2010
Flyers were placed in the community, emails were sent to university staff and Passive

a television advertisement was broadcast. It is not specifically stated who conducted the recruitment.

A three phased sequenced approach was used. Researchers and a research nurse reviewed medical records. Potential participants were sent a letter and recruited during their scheduled visits with the primary health care providers or following an expression of interest. Nurses conducted a pre screening and financial compensation to offset costs of visits to the centre was provided.

Participants were recruited via referral by primary care providers, but which specific type of care provider was not reported. It is not specifically stated who conducted the recruitment.

Researchers recruited participants via workplace email.

Active/Passive

A two phased sequenced approach was used. Researchers marched electoral roll lists against telephone directory lists to identify potential participants who owned phones. A preceding postcard informing the recruit about the study and the likelihood of a phone call to follow. Phone calls were then made by members of the research team and approximately 9 calls were required to recruit one participant.

A three phased sequenced approach was used. Researchers, assisted by staff nurses sent an eligibility questionnaire to a randomly selected group from a GP client list (GP letters included). This was followed by a letter explaining the study to those expressing an interest and then a phone call to the responders to arrange which info session they could attend.

Researchers and trained female students conducted telephone calls, face to face approaches at supermarkets, direct mailing and flyers.

Clinical staff recruited women by letter and phone follow up in two health centres. The paper also states that in another centre clinical populations were recruited, but this is not clearly explained. Women who were also past participants in a case control study and had agreed to take part in future research.

Invitation by proxy during the NSW phone Health Survey. Researchers in this Passive study then produced a community based newspaper and sent intranet messages in the area health services (it is not clear what they meant by that).

Researchers placed newspaper ads and posters on a university campus. Researcher also visited classes on college campus and conducted face to face recruitment on campus.

Researchers placed adverts in a local newspaper and flyers at local hospital, clinics, and other public locations. A listing was placed on a medical research recruitment site. Information and water bottles were given to potential participants and doctors to raise the profile of the study and encourage referrals from doctors.

Researchers used flyers and information meetings.

Passive/Active

Pastors who attended luncheons regarding health promotion and disease Active/Passive prevention strategies among African Americans were recruited to help introduce the intervention and aid recruitment of participants. Following this, researchers placed flyers in churches, bulletins in newsletters, announcements at Sunday services and held information meetings.

Posters and internet ads were sent as part of an employee health Passive programme. It is not specifically stated who conducted the recruitment.

Posters, cards on food hall tables and two community newspapers were used Passive/Active to circulate information. Three presentations were held at local health programme meetings. It is not stated who conducted the recruitment.

Flyers were placed at the local maternity wards, doctors' surgeries, early childhood centres, day care centres, immunization clinics, baby product stores and playgrounds. Adverts placed in school bulletins; local newspapers and also paid adverts in newspapers. Information sessions were conducted for new mothers in childhood centres. It is not specifically stated who conducted the recruitment.

Adverts were placed in local newspapers. Radio interviews were conducted. It Passive 
Table 5 Recruitment planning and implementation (Quality Metric categories) (Continued)

\begin{tabular}{|c|c|c|c|c|}
\hline $\begin{array}{l}\text { 29. De Cocker } \\
\text { et al } 2007\end{array}$ & 3 & $\begin{array}{l}\text { Telephone calls and postal mail invites to } 2500 \text { randomly selected members } \\
\text { of the registered population. A multi-media campaign was carried out to } \\
\text { raise awareness of the programme. It is not specifically stated who } \\
\text { conducted the recruitment. }\end{array}$ & Active/Passive & 2 \\
\hline $\begin{array}{l}\text { 30. Dinger et al, } \\
2005\end{array}$ & 2 & $\begin{array}{l}\text { Emails were sent to university staff and adverts were placed on the University } \\
\text { television station. It is not specifically stated who conducted the recruitment. }\end{array}$ & Passive & 2 \\
\hline $\begin{array}{l}\text { 31. Engel and } \\
\text { Lindner, } 2006\end{array}$ & 1 & $\begin{array}{l}\text { A 'local Media campaign' was conducted. It is not specifically stated who } \\
\text { conducted the recruitment. }\end{array}$ & Passive & 2 \\
\hline $\begin{array}{l}\text { 32. Foreman et } \\
\text { al, } 2001\end{array}$ & 2 & $\begin{array}{l}\text { This qualitative paper did not clearly describe the processes behind their } \\
\text { recruitment approach. It emphasises the need for the walk leaders and } \\
\text { organisers to become actively engaged in the process and how interpersonal } \\
\text { approaches are highly necessary and more effective in engaging a broader } \\
\text { range of participants or specific target groups. }\end{array}$ & Active/Passive & 2 \\
\hline $\begin{array}{l}\text { 33. Humpel et } \\
\text { al, } 2004\end{array}$ & 1 & $\begin{array}{l}\text { Letters were sent to individuals listed in an insurance company client list, } \\
\text { with follow up letters to non-responders. It is not specifically stated who } \\
\text { conducted the recruitment. }\end{array}$ & Passive & 2 \\
\hline $\begin{array}{l}\text { 34. Nies et al, } \\
2006\end{array}$ & 2 & $\begin{array}{l}\text { Flyers were placed in the local community and the programme was } \\
\text { promoted on the radio. It is not specifically stated who conducted the } \\
\text { recruitment. }\end{array}$ & Passive & 2 \\
\hline $\begin{array}{l}\text { 35. Purath et al, } \\
2004\end{array}$ & 1 & $\begin{array}{l}\text { Participants were recruited at annual workplace health screenings. May have } \\
\text { been pre-notified but this isn't stated. It is not specifically stated who } \\
\text { conducted the recruitment. }\end{array}$ & Active & 2 \\
\hline $\begin{array}{l}\text { 36. Shaw et al, } \\
2007\end{array}$ & 4 & $\begin{array}{l}\text { The study was promoted via workplace intranet, staff newsletter and flyers. } \\
\text { Emails were sent to managers of departments to be forwarded to staff. It is } \\
\text { not specifically stated who conducted the recruitment. }\end{array}$ & Passive & 2 \\
\hline $\begin{array}{l}\text { 37. Sidman et } \\
\text { al, } 2004\end{array}$ & 1 & $\begin{array}{l}\text { Flyers were posted on two University campuses. It is not specifically stated } \\
\text { who conducted the recruitment. }\end{array}$ & Passive & 2 \\
\hline $\begin{array}{l}\text { 38. Thomas and } \\
\text { Williams, } 2006\end{array}$ & 1 & $\begin{array}{l}\text { Emails were distributed in the workplace. It is not specifically stated who } \\
\text { conducted the recruitment. }\end{array}$ & Passive & 2 \\
\hline $\begin{array}{l}\text { 39. Tudor-Locke } \\
\text { et al, } 2002\end{array}$ & 1 & $\begin{array}{l}\text { Recruited at/after an diabetes education session. Convenience sample, first } \\
\text { come first serve. It is not specifically stated who conducted the recruitment. }\end{array}$ & Active & 2 \\
\hline $\begin{array}{l}\text { 40. Baker et al, } \\
\text { 2008a }\end{array}$ & 3 & $\begin{array}{l}\text { Posters and newsletters were placed on a University campus. Emails were } \\
\text { sent to University staff. It is not specifically stated who conducted the } \\
\text { recruitment. }\end{array}$ & Passive & 1 \\
\hline $\begin{array}{l}\text { 41. Hultquist et } \\
\text { al, } 2005\end{array}$ & 2 & $\begin{array}{l}\text { Flyers were placed on a University campus and in the surrounding area. The } \\
\text { study was publicised in a local newsletter. It is not specifically stated who } \\
\text { conducted the recruitment. }\end{array}$ & Passive & 1 \\
\hline $\begin{array}{l}\text { 42. Lomabrd et } \\
\text { al, } 1995\end{array}$ & 2 & $\begin{array}{l}\text { Newspaper advertisements and flyers were posted on campus at a University. } \\
\text { It is not specifically stated who conducted the recruitment. }\end{array}$ & Passive & 1 \\
\hline $\begin{array}{l}\text { 43. DNSWH, } \\
2002\end{array}$ & 4 & $\begin{array}{l}\text { Flyers distributed via letter box drop. Use of a 'feature' newspaper article. } \\
\text { Information sent to local community groups (e.g. Rotary and Lions), schools, } \\
\text { preschools, playgroups, community nurses, doctors' surgeries, local rugby } \\
\text { club, and local business (e.g. chemists' shops, real estate agents, car } \\
\text { dealerships). Poster and flyers placed in parks, at bus stops, local streets, } \\
\text { shops, libraries and other public facilities. It is not specifically stated who } \\
\text { conducted the recruitment. }\end{array}$ & Passive & 1 \\
\hline $\begin{array}{l}\text { 44. Rovniak, } \\
2005\end{array}$ & 5 & $\begin{array}{l}\text { The methods are reported as: the use of local list-servs for direct mailing; } \\
\text { churches; a news brief on a local radio and television station, a university } \\
\text { newspaper article, and flyers. It is not specifically stated who conducted the } \\
\text { recruitment. }\end{array}$ & Passive & 1 \\
\hline $\begin{array}{l}\text { 45. Rowley et } \\
\text { al, } 2007\end{array}$ & Unclear & $\begin{array}{l}\text { The paper reports only the following details regarding recruitment: 'There } \\
\text { was an enthusiastic response from invited mothers and many requests to join } \\
\text { from other who had heard about the programme through local publicity and } \\
\text { word of mouth'. It is not specifically stated who conducted the recruitment. }\end{array}$ & Passive/Active & 1 \\
\hline $\begin{array}{l}\text { 46. Talbot et al, } \\
2003\end{array}$ & 2 & $\begin{array}{l}\text { Participants were recruited through senior centres and advertisements in local } \\
\text { newspapers. It is not specifically stated who conducted the recruitment. }\end{array}$ & Passive/Active & 1 \\
\hline $\begin{array}{l}\text { 47. Wyatt et al, } \\
2004\end{array}$ & 1 & $\begin{array}{l}\text { Word of mouth at a 'kick start' session. It is not specifically stated who } \\
\text { conducted the recruitment. }\end{array}$ & Active & 1 \\
\hline
\end{tabular}




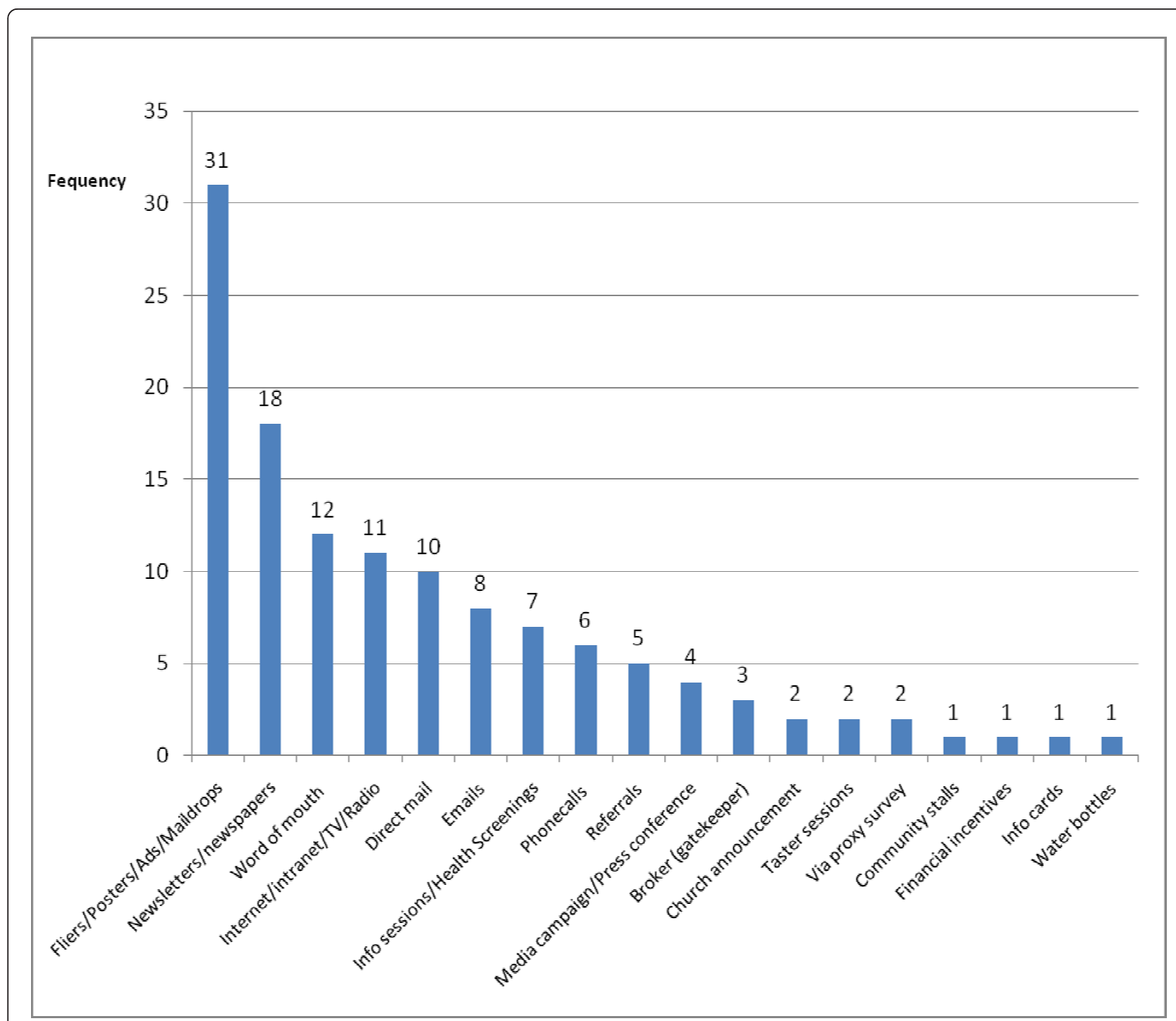

Figure 2 Methods of recruitment and frequency of use from all included studies $(n=47)$.

practice model which details how best to recruit participants as well as what is the best intervention to promote walking. Our conceptual framework offers researchers, practitioners and policy makers a way forward to develop and assess the success of a recruitment strategy to target particular groups. The model offers options through the four stages to assess how many people are responding and engaging in a walking intervention, but also whether any bias is occurring and if efforts need to be refined towards a specific group. It could also provide a true picture of the costs of the intervention as the inclusion of recruitment development and implementation should be included in economic evaluations.

The results of our review could translate into a series of recruitment principles for further evaluation by researchers. These principles include (i) form recruitment plans and strategies on evidence of what the target group feels would be appropriate, based on formative research, (ii) conduct a pilot phase of testing, (iii) recruit in places where the participants are located, (iv) allow sufficient time to recruit participants and monitor the uptake, (v) provide training in recruitment methods for recruitment staff, (vi) monitor the participants response to recruitment approaches and use different recruitment strategies where necessary.

The future of walking and physical activity promotion will lie not only in establishing the effectiveness of different interventions but also in improved recruitment practice. Currently, generalisability is limited by reach within studies; but while the current methods being used are applied, the current limited reach will prevail. We offer principles for recruitment that require further 
Table 6 Settings and Locations of recruitment, study and populations

\begin{tabular}{|c|c|c|c|c|c|c|}
\hline $\begin{array}{l}\text { Study } \\
\text { Author } \\
\text { (Year) }\end{array}$ & $\begin{array}{l}\text { Stated } \\
\text { Study } \\
\text { setting }\end{array}$ & $\begin{array}{l}\text { Target } \\
\text { population }\end{array}$ & $\begin{array}{l}\text { Where did the Recruitment } \\
\text { take place? }\end{array}$ & Intervention delivery site & $\begin{array}{l}\text { Where Participants came } \\
\text { from }\end{array}$ & $\begin{array}{l}\text { Quality } \\
\text { Metric } \\
\text { Score }\end{array}$ \\
\hline $\begin{array}{l}\text { I. Watson } \\
\text { et al, } 2005\end{array}$ & Community & $\begin{array}{l}\text { Post-natal } \\
\text { mothers }\end{array}$ & $\begin{array}{l}\text { Home, health centre visits, at } \\
\text { mothers group meetings }\end{array}$ & Community via lead walks & $\begin{array}{l}\text { Mothers using community } \\
\text { health centres or early } \\
\text { childhood health centres } \\
\text { or mothers visited by local } \\
\text { childcare nurses }\end{array}$ & 5 \\
\hline $\begin{array}{l}\text { 2. Banks- } \\
\text { Wallace et } \\
\text { al, } 2004\end{array}$ & $\begin{array}{l}\text { Community } \\
\text { Setting: } \\
\text { African } \\
\text { American } \\
\text { (AA) }\end{array}$ & $\begin{array}{l}\text { African } \\
\text { American } \\
\text { women in a } \\
\text { local } \\
\text { community } \\
\text { (Minority } \\
\text { group) }\end{array}$ & $\begin{array}{l}\text { In the community at venues } \\
\text { typically used for hosting } \\
\text { African American community } \\
\text { events }\end{array}$ & $\begin{array}{l}\text { Local community venue used } \\
\text { for hosting AA community } \\
\text { events }\end{array}$ & $\begin{array}{l}\text { African American } \\
\text { Community }\end{array}$ & 4 \\
\hline $\begin{array}{l}\text { 3. Kolt et } \\
\text { al, } 2006\end{array}$ & Community & $\begin{array}{l}\text { Older } \\
\text { sedentary } \\
\text { adults }(>65)\end{array}$ & $\begin{array}{l}\text { By mail and a follow up home } \\
\text { visit }\end{array}$ & $\begin{array}{l}\text { By phone and a home visit at } \\
\text { screening (Community) }\end{array}$ & GP Patient lists & 4 \\
\hline $\begin{array}{l}\text { 4. Nguyen } \\
\text { et al, } 2002\end{array}$ & Community & $\begin{array}{l}\text { General } \\
\text { community }\end{array}$ & $\begin{array}{l}\text { Mainly passively in the } \\
\text { community but also used } \\
\text { press conferences and info/ } \\
\text { taster sessions }\end{array}$ & & Community & 4 \\
\hline $\begin{array}{l}5 . \\
\text { Prestwich } \\
\text { et al, } 2010\end{array}$ & University & $\begin{array}{l}\text { University } \\
\text { students }\end{array}$ & University & University & University students & 4 \\
\hline $\begin{array}{l}\text { 6. Rowland } \\
\text { et al, } 2004\end{array}$ & Community & $\begin{array}{l}\text { Sedentary older } \\
\text { adults }\end{array}$ & $\begin{array}{l}\text { Via telephone, direct mail and } \\
\text { then at multiple locations and } \\
\text { media in the community }\end{array}$ & At home & $\begin{array}{l}\text { Community members } \\
\text { identified through a } \\
\text { commercial database of } \\
\text { household data }\end{array}$ & 4 \\
\hline $\begin{array}{l}\text { 7. Sherman } \\
\text { et al, } 2006\end{array}$ & $\begin{array}{l}\text { Community } \\
\text { (Rural) }\end{array}$ & Rural women & $\begin{array}{l}\text { In a clinic, hair salons-and } \\
\text { food establishments }\end{array}$ & Clinical centre & $\begin{array}{l}\text { Residents in the local } \\
\text { community }\end{array}$ & 4 \\
\hline $\begin{array}{l}\text { 8. Wilbur et } \\
\text { al, } 2006\end{array}$ & $\begin{array}{l}\text { Community } \\
\text { and Home }\end{array}$ & $\begin{array}{l}\text { African } \\
\text { American } \\
\text { Women }\end{array}$ & $\begin{array}{l}\text { Two federally qualified } \\
\text { community health centres } \\
\text { serving poor and working } \\
\text { class urban populations. } \\
\text { Screening and data collection } \\
\text { was carried out here to } \\
\text { reduce power differences } \\
\text { (perceived) and increase trust. } \\
\text { Concentrated on an area } \\
\text { within a 3-mile radius of the } \\
\text { data collection sites. Also } \\
\text { interacted in the community } \\
\text { at health fairs and } \\
\text { presentations. }\end{array}$ & $\begin{array}{l}\text { Community health centres. } \\
\text { Purposely chosen to reduce } \\
\text { power differences and } \\
\text { increase trust. Within three } \\
\text { miles of the participants } \\
\text { residential area }\end{array}$ & $\begin{array}{l}\text { Predominantly African } \\
\text { American women within a } \\
\text { 3-mile radius of the } \\
\text { intervention centre }\end{array}$ & 4 \\
\hline $\begin{array}{l}\text { 9. Baker et } \\
\text { al, 2008b }\end{array}$ & Community & $\begin{array}{l}\text { Community } \\
\text { members in } \\
\text { areas of high } \\
\text { deprivation (> } \\
15 \% \text { SIMD) }\end{array}$ & $\begin{array}{l}\text { Local community, GP } \\
\text { surgeries, shops, community } \\
\text { stalls }\end{array}$ & University campus & $\begin{array}{l}\text { Residents within a } \\
\text { surrounding area of West } \\
\text { Glasgow university ( } 1.5 \\
\text { km)-defined as a suitable } \\
\text { walking distance from } \\
\text { intervention site }\end{array}$ & 3 \\
\hline $\begin{array}{l}10 . \\
\text { Brownson } \\
\text { et al } 2005\end{array}$ & $\begin{array}{l}\text { Community } \\
\text { (Rural USA) }\end{array}$ & $\begin{array}{l}\text { Rural } \\
\text { community } \\
\text { members }\end{array}$ & $\begin{array}{l}\text { Through media, at physicians } \\
\text { practices, at community } \\
\text { centres, on walking routes, in } \\
\text { the community active and } \\
\text { passively }\end{array}$ & Community & Within targeted community & 3 \\
\hline $\begin{array}{l}\text { 11. Cox et } \\
\text { al, } 2008\end{array}$ & Community & $\begin{array}{l}\text { Previously } \\
\text { sedentary older } \\
\text { women }\end{array}$ & $\begin{array}{l}\text { Ads delivered in the } \\
\text { community. Screening took } \\
\text { place at the community } \\
\text { centre }\end{array}$ & Community centre & $\begin{array}{l}\text { Recruited from the } \\
\text { community' }\end{array}$ & 3 \\
\hline $\begin{array}{l}\text { 12. Dinger } \\
\text { et al, } 2007\end{array}$ & University & $\begin{array}{l}\text { Insufficiently } \\
\text { active women } \\
\text { (University staff } \\
\text { and local } \\
\text { community } \\
\text { members) }\end{array}$ & Local media and electronically & $\begin{array}{l}\text { Intervention delivered by } \\
\text { email (Virtual) }\end{array}$ & $\begin{array}{l}\text { University staff and local } \\
\text { community }\end{array}$ & 3 \\
\hline
\end{tabular}


Table 6 Settings and Locations of recruitment, study and populations (Continued)

\begin{tabular}{|c|c|c|c|c|c|c|}
\hline $\begin{array}{l}13 . \\
\text { Dubbert et } \\
\text { al, } 2002\end{array}$ & $\begin{array}{l}\text { Care setting } \\
\text { (Veterans } \\
\text { Affairs } \\
\text { Medical } \\
\text { Centre) }\end{array}$ & $\begin{array}{l}\text { Elderly primary } \\
\text { care patients }\end{array}$ & $\begin{array}{l}\text { By mail, phone and at the } \\
\text { clinic }\end{array}$ & Medical centre & $\begin{array}{l}\text { Attendees at a Veterans } \\
\text { Affairs Medical centre }\end{array}$ & 3 \\
\hline $\begin{array}{l}14 . \\
\text { Dubbert et } \\
\text { al, } 2008\end{array}$ & Care setting & Elderly veterans & $\begin{array}{l}\text { Primary care medical centre as } \\
\text { part of routine care }\end{array}$ & Primary care clinic & $\begin{array}{l}\text { Primary care clinics for } \\
\text { veterans }\end{array}$ & 3 \\
\hline $\begin{array}{l}\text { 15. Gilson } \\
\text { et al, } 2008\end{array}$ & $\begin{array}{l}\text { Workplace } \\
\text { (University) }\end{array}$ & $\begin{array}{l}\text { Work-place } \\
\text { employees }\end{array}$ & Via work email & University & University employees & 3 \\
\hline $\begin{array}{l}\text { 16. Jancey } \\
\text { et al, } 2008\end{array}$ & Community & Older adults & $\begin{array}{l}\text { Over the phone to home } \\
\text { phone numbers }\end{array}$ & $\begin{array}{l}\text { Selected green space areas } \\
\text { within the neighbourhood } \\
\text { local to the recruited } \\
\text { participants }\end{array}$ & $\begin{array}{l}\text { Urban areas of Perth, } \\
\text { identified through electoral } \\
\text { roll }\end{array}$ & 3 \\
\hline $\begin{array}{l}\text { 17. Lamb } \\
\text { et al, } 2002\end{array}$ & $\begin{array}{l}\text { Care (Primary } \\
\text { care) }\end{array}$ & $\begin{array}{l}\text { Middle aged } \\
\text { adults }\end{array}$ & $\begin{array}{l}\text { Via post, phone and info } \\
\text { sessions at primary care } \\
\text { setting }\end{array}$ & Primary care facilities & Primary care client list & 3 \\
\hline $\begin{array}{l}\text { 18. Lee et } \\
\text { al, } 1997\end{array}$ & Community & $\begin{array}{l}\text { Sedentary } \\
\text { ethnic minority } \\
\text { women }\end{array}$ & $\begin{array}{l}\text { Directly and indirectly in the } \\
\text { community }\end{array}$ & $\begin{array}{l}\text { Baseline screening at a } \\
\text { University, then indirectly } \\
\text { delivered to participants } \\
\text { homes }\end{array}$ & $\begin{array}{l}\text { Members of women, } \\
\text { children and infant groups, } \\
\text { local area San Diego }\end{array}$ & 3 \\
\hline $\begin{array}{l}19 . \\
\text { Matthews } \\
\text { et al, } 2007\end{array}$ & $\begin{array}{l}\text { Care: Clinical } \\
\text { and Home } \\
\text { (Community) } \\
\text { setting }\end{array}$ & $\begin{array}{l}\text { Breast cancer } \\
\text { survivors }\end{array}$ & Clinic & Clinical centres & $\begin{array}{l}\text { Former or existing clinical } \\
\text { populations }\end{array}$ & 3 \\
\hline $\begin{array}{l}\text { 20. Merom } \\
\text { et al, } 2007\end{array}$ & Community & Inactive adults & $\begin{array}{l}\text { Passively in the community } \\
\text { and actively by phone via } \\
\text { another study }\end{array}$ & $\begin{array}{l}\text { This was a passively delivered } \\
\text { intervention and participants } \\
\text { received intervention material } \\
\text { and equipment entirely by } \\
\text { post. }\end{array}$ & $\begin{array}{l}\text { Non-clinical sample of } \\
\text { individuals in the } \\
\text { community }\end{array}$ & 3 \\
\hline $\begin{array}{l}\text { 21. Ornes } \\
\text { and } \\
\text { Ransdell, } \\
2007\end{array}$ & University & Women & University campus & University & University & 3 \\
\hline $\begin{array}{l}22 . \\
\text { Richardson } \\
\text { et al, } 2007\end{array}$ & Care: Clinical & $\begin{array}{l}\text { Adults with } \\
\text { type } 2 \text { diabetes }\end{array}$ & Medical centre & Clinical centre & $\begin{array}{l}\text { Adults with diabetes living } \\
\text { in the community }\end{array}$ & 3 \\
\hline $\begin{array}{l}23 . \\
\text { Rosenberg } \\
\text { et al, } 2009\end{array}$ & $\begin{array}{l}\text { Care setting } \\
\text { (Retirement } \\
\text { community) }\end{array}$ & Older adults & Care community & $\begin{array}{l}\text { Continuing care retirement } \\
\text { community }\end{array}$ & Residential care facility & 3 \\
\hline $\begin{array}{l}\text { 24. Whitt- } \\
\text { Glover et } \\
\text { al, } 2008\end{array}$ & Churches & $\begin{array}{l}\text { Black adult, } \\
\text { church } \\
\text { attendees }\end{array}$ & $\begin{array}{l}\text { University and Local } \\
\text { Community }\end{array}$ & Church meeting rooms & Church groups & 3 \\
\hline $\begin{array}{l}\text { 25. Arbour } \\
\& \text { Ginis, } \\
2009\end{array}$ & Workplace & $\begin{array}{l}\text { Women in the } \\
\text { workplace }\end{array}$ & University campus & Workplace (University) & University & 2 \\
\hline $\begin{array}{l}\text { 26. Culos- } \\
\text { Reed et al, } \\
2008\end{array}$ & $\begin{array}{l}\text { Community: } \\
\text { Malls }\end{array}$ & NS & $\begin{array}{l}\text { In the community and at the } \\
\text { malls }\end{array}$ & Mall & $\begin{array}{l}\text { Mall users from the local } \\
\text { community }\end{array}$ & 2 \\
\hline $\begin{array}{l}\text { 27. Currie } \\
\text { and } \\
\text { Develin, } \\
2001\end{array}$ & Community & $\begin{array}{l}\text { Mothers and } \\
\text { young children }\end{array}$ & $\begin{array}{l}\text { Places where pre and post } \\
\text { natal mums engage with } \\
\text { health care, shopping and } \\
\text { school }\end{array}$ & Community & NS & 2 \\
\hline $\begin{array}{l}\text { 28. Darker } \\
\text { et al, } 2010\end{array}$ & $\begin{array}{l}\text { Clinical lab } \\
\text { setting }\end{array}$ & NS & In the local media (Passive) & Laboratory & NS & 2 \\
\hline $\begin{array}{l}\text { 29. De } \\
\text { Cocker et } \\
\text { al } 2007\end{array}$ & Community & $\begin{array}{l}\text { 'General } \\
\text { population' } \\
\text { adults in a } \\
\text { local } \\
\text { community }\end{array}$ & $\begin{array}{l}\text { By mail or phone to } \\
\text { participants homes. Indirect } \\
\text { but active }\end{array}$ & $\begin{array}{l}\text { In the community with } \\
\text { contact via phone and mail } \\
\text { for pedometer packs }\end{array}$ & $\begin{array}{l}\text { General population } \\
\text { members as listed on the } \\
\text { population register }\end{array}$ & 2 \\
\hline
\end{tabular}


Table 6 Settings and Locations of recruitment, study and populations (Continued)

\begin{tabular}{|c|c|c|c|c|c|c|}
\hline $\begin{array}{l}\text { 30. Dinger } \\
\text { et al, } 2005\end{array}$ & University & $\begin{array}{l}\text { Female } \\
\text { employees or } \\
\text { spouses of } \\
\text { university } \\
\text { employees }\end{array}$ & University & University campus & University staff and spouses & 2 \\
\hline $\begin{array}{l}\text { 31. Engel } \\
\text { and } \\
\text { Lindner, } \\
2006\end{array}$ & Community & $\begin{array}{l}\text { Adults with } \\
\text { type } 2 \text { diabetes }\end{array}$ & In community via newspapers & $\begin{array}{l}\text { At research institute or at } \\
\text { home }\end{array}$ & Local Community & 2 \\
\hline $\begin{array}{l}32 . \\
\text { Foreman et } \\
\text { al, } 2001\end{array}$ & Community & $\begin{array}{l}\text { Community } \\
\text { members }\end{array}$ & NS & NS & NS & 2 \\
\hline $\begin{array}{l}\text { 33. Humpel } \\
\text { et al, } 2004\end{array}$ & Community & $\begin{array}{l}\text { Over } 40 \text { year } \\
\text { old community } \\
\text { members }\end{array}$ & Via post. No face to face & $\begin{array}{l}\text { No face to face contact, but } \\
\text { participants encouraged to } \\
\text { walk in their local area }\end{array}$ & $\begin{array}{l}\text { Insurance company client } \\
\text { list }\end{array}$ & 2 \\
\hline $\begin{array}{l}\text { 34. Nies et } \\
\text { al, } 2006\end{array}$ & Community & $\begin{array}{l}\text { European } \\
\text { American and } \\
\text { African America } \\
\text { women. }\end{array}$ & $\begin{array}{l}\text { Through media and fliers in } \\
\text { the community }\end{array}$ & NS & NS & 2 \\
\hline $\begin{array}{l}\text { 35. Purath } \\
\text { et al, } 2004\end{array}$ & $\begin{array}{l}\text { Workplace } \\
\text { (University) }\end{array}$ & $\begin{array}{l}\text { Women in the } \\
\text { workplace }\end{array}$ & $\begin{array}{l}\text { Health screening day within a } \\
\text { university }\end{array}$ & University & $\begin{array}{l}\text { Staff attending a voluntary } \\
\text { university provided health } \\
\text { screening as part of a } \\
\text { wellness programme }\end{array}$ & 2 \\
\hline $\begin{array}{l}\text { 36. Shaw et } \\
\text { al, } 2007\end{array}$ & $\begin{array}{l}\text { Workplace } \\
\text { (Health } \\
\text { Centre staff) }\end{array}$ & $\begin{array}{l}\text { Men and } \\
\text { women in the } \\
\text { workplace }\end{array}$ & Workplace (Health centre) & Workplace (Urban workplace) & Health Centre staff & 2 \\
\hline $\begin{array}{l}\text { 37. Sidman } \\
\text { et al, } 2004\end{array}$ & $\begin{array}{l}\text { University } \\
\text { (Seems Uni) }\end{array}$ & $\begin{array}{l}\text { Sedentary } \\
\text { women }\end{array}$ & Two University campuses & NS & $\begin{array}{l}\text { NS (Recruited on Uni } \\
\text { campus) }\end{array}$ & 2 \\
\hline $\begin{array}{l}\text { 38. Thomas } \\
\text { and } \\
\text { Williams, } \\
2006\end{array}$ & Workplace & $\begin{array}{l}\text { Workplace staff } \\
\text { (Excluding } \\
\text { hospital and } \\
\text { community } \\
\text { services staff) }\end{array}$ & Workplace (Electronically) & NS & $\begin{array}{l}\text { Workplace staff (Dept. of } \\
\text { Human Services staff) }\end{array}$ & 2 \\
\hline $\begin{array}{l}\text { 39. Tudor- } \\
\text { Locke et al, } \\
2002\end{array}$ & Health centre & $\begin{array}{l}\text { Sedentary } \\
\text { diabetes } \\
\text { sufferers }\end{array}$ & Diabetes Centre & Diabetes care centre & Diabetes care centre & 2 \\
\hline $\begin{array}{l}\text { 40. Baker et } \\
\text { al, 2008a }\end{array}$ & University & NS & At churches & University campus & University campus & 1 \\
\hline $\begin{array}{l}41 . \\
\text { Hultquist et } \\
\text { al, } 2005\end{array}$ & University & NS & University & University & University campus & 1 \\
\hline $\begin{array}{l}42 . \\
\text { Lomabrd } \\
\text { et al, } 1995\end{array}$ & University & NS & University campus & University & University staff & 1 \\
\hline $\begin{array}{l}43 . \\
\text { DNSWH, } \\
2002\end{array}$ & Community & NS & $\begin{array}{l}\text { In local area via media and } \\
\text { advertising and information }\end{array}$ & Community & $\begin{array}{l}\text { Residents of local } \\
\text { community }\end{array}$ & 1 \\
\hline $\begin{array}{l}\text { 44. Rovniak, } \\
2005\end{array}$ & Community & NS & $\begin{array}{l}\text { At multiple locations in the } \\
\text { community. Mainly passive. }\end{array}$ & NS & NS (Seems community) & 1 \\
\hline $\begin{array}{l}\text { 45. Rowley } \\
\text { et al, } 2007\end{array}$ & Community & $\begin{array}{l}\text { Parents and } \\
\text { children }\end{array}$ & NS & $\begin{array}{l}\text { In the community along } \\
\text { planned walking routes in } \\
\text { and out of parks/green spaces }\end{array}$ & $\begin{array}{l}\text { Affluent community in } \\
\text { semi-rural England' }\end{array}$ & 1 \\
\hline $\begin{array}{l}\text { 46. Talbot } \\
\text { et al, } 2003\end{array}$ & $\begin{array}{l}\text { Community } \\
\text { (Home) }\end{array}$ & Older adults & $\begin{array}{l}\text { Senior centres, ads in local } \\
\text { newspapers }\end{array}$ & University clinic & Local Community & 1 \\
\hline $\begin{array}{l}\text { 47. Wyatt } \\
\text { et al, } 2004\end{array}$ & Community & $\begin{array}{l}\text { State wide } \\
\text { residents of the } \\
\text { community }\end{array}$ & NS & $\begin{array}{l}\text { Worksite and Church via a } \\
\text { starter kit }\end{array}$ & Workplaces and church & 1 \\
\hline
\end{tabular}


Table 7 Recruitment rates and efficiency ratios

\begin{tabular}{|c|c|c|c|c|c|c|c|c|c|c|}
\hline $\begin{array}{l}\text { Study } \\
\text { Author } \\
\text { (Year) }\end{array}$ & Pool & Invited & Responded & Started & $\begin{array}{l}\text { Efficiency A } \\
\text { (\%) (Started/ } \\
\text { Pool) }\end{array}$ & $\begin{array}{l}\text { Efficiency B } \\
\text { (\%) (Started/ } \\
\text { Invited) }\end{array}$ & $\begin{array}{l}\text { Efficiency C (\%) } \\
\text { (Started/ } \\
\text { Responded) }\end{array}$ & $\begin{array}{l}\text { Efficiency } \\
\mathrm{D}(\mathrm{N}) \\
\text { (Started } \\
\text { only) }\end{array}$ & $\begin{array}{l}\text { Weekly } \\
\text { Recruitment } \\
\text { Rate }\end{array}$ & $\begin{array}{c}\text { Quality } \\
\text { Metric } \\
\text { Score }\end{array}$ \\
\hline $\begin{array}{l}\text { I. Watson et } \\
\text { al, } 2005\end{array}$ & NS & NS & NS & 139 & - & - & & 139.0 & 23.17 & 5 \\
\hline $\begin{array}{l}\text { 2. Banks- } \\
\text { Wallace et al, } \\
2004\end{array}$ & NS & NS & 38 & 21 & - & - & 55.3 & 21.0 & 0.97 & 4 \\
\hline $\begin{array}{l}\text { 3. Kolt et al, } \\
2006\end{array}$ & NS & NS & NS & 186 & - & - & - & 186.0 & 4.77 & 4 \\
\hline $\begin{array}{l}\text { 4. Nguyen et } \\
\text { al, } 2002\end{array}$ & NS & NS & NS & NS & - & - & - & NS & & 4 \\
\hline $\begin{array}{l}\text { 5. Prestwich } \\
\text { et al, } 2010\end{array}$ & NS & NS & 173 & 149 & - & - & 86.1 & 149.0 & 59.60 & 4 \\
\hline $\begin{array}{l}\text { 6. Rowland et } \\
\text { al, } 2004\end{array}$ & 73828 & NS & NS & 582 & 0.8 & - & - & 582.0 & 13.44 & 4 \\
\hline $\begin{array}{l}\text { 7. Sherman et } \\
\text { al, } 2006\end{array}$ & 1700 & NS & 75 & 75 & 4.4 & - & 100.0 & 75.0 & 267.86 & 4 \\
\hline $\begin{array}{l}\text { 8. Wilbur et } \\
\text { al, } 2006\end{array}$ & NS & NS & NS & 281 & - & - & - & 281.0 & 2.32 & 4 \\
\hline $\begin{array}{l}\text { 9. Baker et al, } \\
2008 \mathrm{~b}\end{array}$ & NS & NS & 169 & 80 & - & - & 47.3 & 80.0 & 3.70 & 3 \\
\hline $\begin{array}{l}\text { 10. Brownson } \\
\text { et al } 2005\end{array}$ & NS & NS & NS & NS & - & - & - & - & & 3 \\
\hline $\begin{array}{l}\text { 11. Cox et al, } \\
2008\end{array}$ & NS & NS & 1312 & 124 & - & - & 9.5 & 124.0 & & 3 \\
\hline $\begin{array}{l}\text { 12. Dinger et } \\
\text { al, } 2007\end{array}$ & NS & NS & 87 & 74 & - & - & 85.1 & 74.0 & 17.21 & 3 \\
\hline $\begin{array}{l}\text { 13. Dubbert } \\
\text { et al, } 2002\end{array}$ & 576 & 576 & 253 & 212 & 36.8 & 36.8 & 83.8 & 212.0 & & 3 \\
\hline $\begin{array}{l}\text { 14. Dubbert } \\
\text { et al, } 2008\end{array}$ & 572 & 572 & NS & 224 & 39.2 & 39.2 & - & 224.0 & & 3 \\
\hline $\begin{array}{l}\text { 15. Gilson et } \\
\text { al, } 2008\end{array}$ & NS & NS & 102 & 70 & - & - & 68.6 & 70.0 & & 3 \\
\hline $\begin{array}{l}\text { 16. Jancey et } \\
\text { al, } 2008\end{array}$ & NS & 7378 & NS & 260 & - & 3.5 & - & 260.0 & & 3 \\
\hline $\begin{array}{l}\text { 17. Lamb et } \\
\text { al, } 2002\end{array}$ & 26500 & 2000 & 960 & 260 & 1.0 & 13.0 & 27.1 & 260.0 & & 3 \\
\hline $\begin{array}{l}\text { 18. Lee et al, } \\
1997\end{array}$ & NS & NS & 387 & 128 & - & - & 33.1 & 128.0 & & 3 \\
\hline $\begin{array}{l}\text { 19. Matthews } \\
\text { et al, } 2007\end{array}$ & 117 & 117 & 102 & 36 & 30.8 & 30.8 & 35.3 & 36.0 & & 3 \\
\hline $\begin{array}{l}\text { 20. Merom et } \\
\text { al, } 2007\end{array}$ & NS & NS & 692 & 369 & - & - & 53.3 & 369.0 & & 3 \\
\hline $\begin{array}{l}\text { 21. Ornes and } \\
\text { Ransdell, } 2007\end{array}$ & NS & NS & 210 & 121 & - & - & 57.6 & 121.0 & & 3 \\
\hline $\begin{array}{l}22 . \\
\text { Richardson et } \\
\text { al, } 2007\end{array}$ & NS & NS & 76 & 35 & - & - & 46.1 & 35.0 & & 3 \\
\hline $\begin{array}{l}\text { 23. Rosenberg } \\
\text { et al, } 2009\end{array}$ & 400 & 400 & NS & 22 & 5.5 & 5.5 & - & 22.0 & & 3 \\
\hline $\begin{array}{l}\text { 24. Whitt- } \\
\text { Glover et al, } \\
2008\end{array}$ & NS & NS & NS & 87 & - & - & - & 87.0 & & 3 \\
\hline $\begin{array}{l}\text { 25. Arbour \& } \\
\text { Ginis, } 2009\end{array}$ & NS & NS & 129 & 75 & - & - & 58.1 & 75.0 & & 2 \\
\hline $\begin{array}{l}\text { 26. Culos- } \\
\text { Reed et al, } \\
2008\end{array}$ & NS & NS & 87 & 52 & - & - & 59.8 & 52.0 & 26.00 & 2 \\
\hline $\begin{array}{l}\text { 27. Currie and } \\
\text { Develin, } 2001\end{array}$ & NS & NS & 110 & NS & - & - & - & NS & & 2 \\
\hline $\begin{array}{l}\text { 28. Darker et } \\
\text { al, } 2010\end{array}$ & NS & NS & 176 & 132 & - & - & 75.0 & 132.0 & 4.36 & 2 \\
\hline
\end{tabular}


Table 7 Recruitment rates and efficiency ratios (Continued)

\begin{tabular}{|c|c|c|c|c|c|c|c|c|c|}
\hline $\begin{array}{l}\text { 29. De Cocker } \\
\text { et al } 2007\end{array}$ & 5000 & 4065 & NS & 1674 & 33.5 & 41.2 & - & 1674.0 & 2 \\
\hline $\begin{array}{l}\text { 30. Dinger et } \\
\text { al, } 2005\end{array}$ & NS & NS & 43 & 36 & - & - & 83.7 & 36.0 & 2 \\
\hline $\begin{array}{l}\text { 31. Engel and } \\
\text { Lindner, } 2006\end{array}$ & NS & NS & NS & 57 & - & - & - & 57.0 & 2 \\
\hline $\begin{array}{l}\text { 32. Foreman } \\
\text { et al, } 2001\end{array}$ & NS & NS & NS & NS & - & - & - & NS & 2 \\
\hline $\begin{array}{l}\text { 33. Humpel } \\
\text { et al, } 2004\end{array}$ & NS & 982 & 429 & 399 & - & 40.6 & 93.0 & 399.0 & 2 \\
\hline $\begin{array}{l}\text { 34. Nies et al, } \\
2006\end{array}$ & NS & NS & 313 & 253 & - & - & 80.8 & 253.0 & 2 \\
\hline $\begin{array}{l}\text { 35. Purath et } \\
\text { al, } 2004\end{array}$ & NS & NS & NS & 287 & - & - & - & 287.0 & 2 \\
\hline $\begin{array}{l}\text { 36. Shaw et } \\
\text { al, } 2007\end{array}$ & NS & NS & NS & 35 & - & - & - & 35.0 & 2 \\
\hline $\begin{array}{l}\text { 37. Sidman et } \\
\text { al, } 2004\end{array}$ & NS & NS & NS & 114 & - & - & - & 114.0 & 2 \\
\hline $\begin{array}{l}\text { 38. Thomas } \\
\text { and Williams, } \\
2006\end{array}$ & 3500 & NS & 1195 & 1195 & 34.1 & - & 100.0 & 1195.0 & 2 \\
\hline $\begin{array}{l}\text { 39. Tudor- } \\
\text { Locke et al, } \\
2002\end{array}$ & NS & 9 & 9 & 9 & - & 100.0 & 100.0 & 9.0 & 2 \\
\hline $\begin{array}{l}\text { 40. Baker et } \\
\text { al, 2008a }\end{array}$ & NS & NS & 61 & 52 & - & - & 85.2 & 52.0 & 1 \\
\hline $\begin{array}{l}\text { 41. Hultquist } \\
\text { et al, } 2005\end{array}$ & NS & NS & 73 & 58 & - & - & 79.5 & 58.0 & 1 \\
\hline $\begin{array}{l}\text { 42. Lomabrd } \\
\text { et al, } 1995\end{array}$ & 5000 & NS & NS & 135 & 2.7 & - & - & 135.0 & 1 \\
\hline $\begin{array}{l}\text { 43. DNSWH, } \\
2002\end{array}$ & NS & NS & NS & NS & - & - & - & NS & 1 \\
\hline $\begin{array}{l}\text { 44. Rovniak, } \\
2005\end{array}$ & NS & NS & 235 & 65 & - & - & 27.7 & 65.0 & 1 \\
\hline $\begin{array}{l}\text { 45. Rowley et } \\
\text { al, } 2007\end{array}$ & NS & NS & NS & 165 & - & - & - & 165.0 & 1 \\
\hline $\begin{array}{l}\text { 46. Talbot et } \\
\text { al, } 2003\end{array}$ & NS & NS & 64 & 40 & - & - & 62.5 & 40.0 & 1 \\
\hline $\begin{array}{l}\text { 47. Wyatt et } \\
\text { al, } 2004\end{array}$ & NS & NS & 735 & 735 & - & - & 100.0 & 735.0 & 1 \\
\hline
\end{tabular}

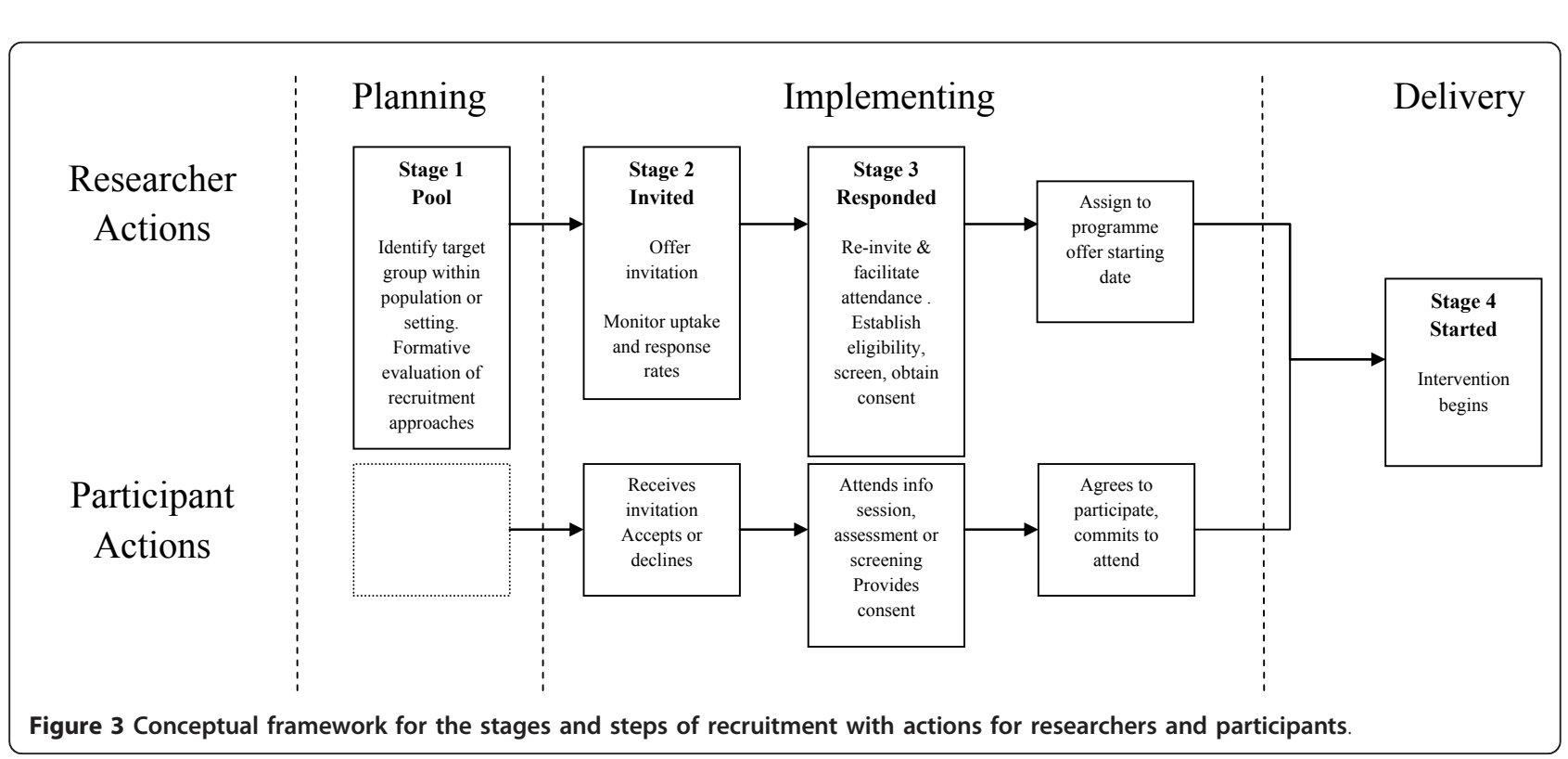


evaluation, (i.e. matching "where to where" and "who to who"). Future research to identify "what is effective recruitment?" may best lie in identifying approaches that reflect the needs and expectations of hard to recruit target groups. This will allow researchers the opportunity to investigate the strategic use of the right recruitment methods, for the right group, in the right order.

\section{Acknowledgements \& funding}

We would like to thank the participants and researches who gave their time to provide our data. NM is the principle investigator for The Scottish Physical Activity Research Collaboration (SPARColl) is based at the University of Strathclyde. The collaboration is funded by the Scottish Government and managed by NHS Health Scotland.

The authors would like to acknowledge the SPARColl advisory group for feedback during the study design and manuscript preparation stage. GB's $\mathrm{PhD}$ is funded by SPARColl. CF is funded by the British Heart Foundation Project Grant (BHF/PG/03/045).

\section{Author details}

'Department of Public Health, University of Oxford, UK. ${ }^{2}$ Scottish Physical Activity Research Collaboration (SPARColl) \& School of Psychological Sciences and Health, University of Strathclyde, Glasgow, UK.

\section{Authors' contributions}

CF, GB \& NM conceived of the study, and participated in its design, coordination and helped to draft the manuscript. CFtz \& CMCA participated in its design and coordination and helped to draft the manuscript. CF, GB \& AM participated in review screening, data extraction and helped to draft the manuscript. All authors read and approved the final manuscript.

\section{Competing interests}

The authors declare that they have no competing interests.

Received: 20 May 2011 Accepted: 15 December 2011

Published: 15 December 2011

\section{References}

1. Hardman AE, Morris JN: Walking to health. Sports Med 1997, 23:306-332

2. Global Advocacy Council for Physical Activity ISfPAaH: The Toronto Charter for Physical Activity: A Global Call for Action. J Phys Act Health 2010, 7(Suppl 3):S370-385.

3. Committee PAGA: Physical Activity Guidelines Advisory Committee Report, 2008 Washington, DC: US Department of Health and Human Services; 2008.

4. Lee IM, Skerrett PJ: Physical activity and all-cause mortality: what is the dose-response relation? Med Sci Sports Exerc 2001, 33:S459-471, discussion S493-454.

5. Murphy MH, Nevill AM, Murtagh EM, Holder RL: The effect of walking on fitness, fatness and resting blood pressure: a meta-analysis of randomised, controlled trials. Prev Med 2007, 44:377-385.

6. Hu FB, Sigal RJ, Rich-Edwards JW, Colditz GA, Solomon CG, Willett WC Speizer FE, Manson JE: Walking compared with vigorous physical activity and risk of type 2 diabetes in women: a prospective study. Jama 1999, 282:1433-1439.

7. Department of Health PA, Health Improvement and Prevention: At least five a week: Evidence on the impact of physical activity and its relationship to health. A report from the Chief Medical Officer. Book At least five a week: Evidence on the impact of physical activity and its relationship to health. A report from the Chief Medical Officer City; 2004, ed. ^eds.

8. MIND: The MIND guide to physical activity London: MIND; 2008.

9. Hamer $\mathrm{M}$, Chida Y: Walking and primary prevention: a meta-analysis of prospective cohort studies. Br J Sports Med 2008, 42:238-243.

10. Ogilvie D, Foster CE, Rothnie H, Cavill N, Hamilton V, Fitzsimons CF, Mutrie N: Interventions to promote walking: systematic review. BMJ 2007, 334:1204.
11. Excellence NIfHaC: Promoting and creating built or natural environments that encourage and support physical activity (NICE public health guidance 8) London: NICE; 2008.

12. Excellence NIfHaC: Promoting physical activity for children and young people London: NICE; 2009.

13. Bauman $\mathrm{A}$, Bull $\mathrm{F}$, Chey $\mathrm{T}$, Craig $\mathrm{CL}$, Ainsworth BE, Sallis JF, Bowles HR, Hagstromer M, Sjostrom M, Pratt M: The International Prevalence Study on Physical Activity: results from 20 countries. Int I Behav Nutr Phys Act 2009, 6:21.

14. Centre TI: Health Survey for England 2008: Physical activity and fitness. Summary of key findings. Book Health Survey for England 2008: Physical activity and fitness. Summary of key findings City; 2009, ed.^eds.

15. Executive S: The Scottish Health Survey 2008 Edinburgh: The Stationery House; 2008

16. Transport Df: Transport Statistics Bulletin: National Travel Survey 2008. Book Transport Statistics Bulletin: National Travel Survey 2008 City: Department for Transport; 2009, ed.^eds.

17. Glasgow RE, Vogt TM, Boles SM: Evaluating the public health impact of health promotion interventions: the RE-AIM framework. Am J Public Health 1999, 89:1322-1327.

18. Schulz KF, Altman DG, Moher D: CONSORT 2010 statement: updated guidelines for reporting parallel group randomised trials. BMJ 2010, 340 c332.

19. Swanson GM, Ward AJ: Recruiting minorities into clinical trials: toward a participant-friendly system. J Natl Cancer Inst 1995, 87:1747-1759.

20. Froelicher ES, Lorig K: Who cares about recruitment anyway? Patient Educ Couns 2002, 48:97.

21. Treweek S, Pitkethly M, Cook J, Kjeldstrom M, Taskila T, Johansen M, Sullivan F, Wilson S, Jackson C, Jones R, Mitchell E: Strategies to improve recruitment to randomised controlled trials. Cochrane Database Syst Rev 2010, MR000013

22. Toerien M, Brookes ST, Metcalfe C, de Salis I, Tomlin Z, Peters TJ, Sterne J, Donovan $J$ : A review of reporting of participant recruitment and retention in RCTs in six major journals. Trials 2009, 10:52.

23. Hillsdon M, Foster C, Thorogood M: Interventions for promoting physical activity. Cochrane Database Syst Rev 2005, CD003180

24. Moher D, Cook DJ, Eastwood S, Olkin I, Rennie D, Stroup DF: Improving the quality of reports of meta-analyses of randomised controlled trials: the QUOROM statement. Quality of Reporting of Meta-analyses. Lancet 1999, 354:1896-1900

25. Excellence NIfHaC: Methods for the development of NICE public health guidance (second edition) London: NICE; 2009.

26. Jadad A: Randomised controlled trials; A users guide London: BMJ Books; 1998.

27. Banks-Wallace J, Enyart J, Johnson C: Recruitment and entrance of participants into a physical activity intervention for hypertensive African American women. ANS Adv Nurs Sci 2004, 27:102-116.

28. Rowland RM, Fishera JK, Greena M, Dunnb AM, Pickeringa MA, Lia F: Recruiting inactive older adults to a neighborhood walking trial: The SHAPE project. Journal of Aging Studies 2004, 18:353-368.

29. Sherman BJ, Gilliland G, Speckman JL, Freund KM: The effect of a primary care exercise intervention for rural women. Prev Med 2007, 44:198-201.

30. Wilbur J, McDevitt JH, Wang E, Dancy BL, Miller AM, Briller J, Ingram DL, Nicola TL, Ju S, Lee H: Outcomes of a home-based walking program for African-American women. Am J Health Promot 2008, 22:307-317.

31. Brownson RC, Hagood L, Lovegreen SL, Britton B, Caito NM, Elliott MB, Emery J, Haire-Joshu D, Hicks D, Johnson B, et al: A multilevel ecological approach to promoting walking in rural communities. Prev Med 2005, 41:837-842.

32. Dinger MK, Heesch $K C$, Cipriani $G$, Qualls $M$ : Comparison of two emaildelivered, pedometer-based interventions to promote walking among insufficiently active women. J Sci Med Sport 2007, 10:297-302.

33. Dubbert PM, Cooper KM, Kirchner KA, Meydrech EF, Billbrew D: Effects of nurse counseling on walking for exercise in elderly primary care patients. J Gerontol A Biol Sci Med Sci 2002, 57:M733-740.

34. Dubbert PM, Morey MC, Kirchner KA, Meydrech EF, Grothe K: Counseling for home-based walking and strength exercise in older primary care patients. Arch Intern Med 2008, 168:979-986

35. Lee RE, McGinnis KA, Sallis JF, Castro CM, Chen AH, Hickmann SA: Active vs. passive methods of recruiting ethnic minority women to a health promotion program. Ann Behav Med 1997, 19:378-384. 
36. Matthews CE, Wilcox S, Hanby CL, Der Ananian C, Heiney SP, Gebretsadik T, Shintani A: Evaluation of a 12-week home-based walking intervention for breast cancer survivors. Support Care Cancer 2007, 15:203-211.

37. Ornes LL, Ransdell LB: Web-Based Physical Activity Intervention for College-Aged Women. Percept Mot Skills 2007, 10:126-137.

38. Richardson CR, Mehari KS, Mclntyre LG, Janney AW, Fortlage LA, Sen A, Strecher VJ, Piette JD: A randomized trial comparing structured and lifestyle goals in an internet-mediated walking program for people with type 2 diabetes. Int I Behav Nutr Phys Act 2007, 4:59.

39. Rosenberg D, Kerr J, Sallis JF, Patrick K, Moore DJ, King A: Feasibility and outcomes of a multilevel place-based walking intervention for seniors: a pilot study. Health Place 2009, 15:173-179.

40. Whitt-Glover MC, Hogan PE, Lang W, Heil DP: Pilot study of a faith-based physical activity program among sedentary blacks. Prev Chronic Dis 2008, 5:A51.

41. Dinger MK, Heesch KC, McClary KR: Feasibility of a minimal contact intervention to promote walking among insufficiently active women. Am $J$ Health Promot 2005, 20:2-6.

42. Nies MA, Partridge T: Comparison of 3 interventions to increase walking in sedentary women. Am J Health Behav 2006, 30:339-352.

43. Purath J, Miller AM, McCabe G, Wilbur J: A brief intervention to increase physical activity in sedentary working women. Can J Nurs Res 2004, 36:76-91.

44. Sidman $\mathrm{CL}$, Corbin $\mathrm{CB}$, Le Masurier $\mathrm{G}$ : Promoting physical activity among sedentary women using pedometers. Res Q Exerc Sport 2004, 75:122-129.

45. Tudor-Locke CE, Myers AM, Bell RC, Harris SB, Wilson Rodger N: Preliminary outcome evaluation of the First Step Program: a daily physical activity intervention for individuals with type 2 diabetes. Patient Educ Couns 2002, 47:23-28.

46. Hultquist CN, Albright C, Thompson DL: Comparison of walking recommendations in previously inactive women. Med Sci Sports Exerc 2005, 37:676-683.

47. Lombard DN, Lombard TN, Winett RA: Walking to meet health guidelines: the effect of prompting frequency and prompt structure. Health Psychol 1995, 14:164-170.

48. Rovniak LS, Hovell MF, Wojcik JR, Winett RA, Martinez-Donate AP: Enhancing theoretical fidelity: an e-mail-based walking program demonstration. Am J Health Promot 2005, 20:85-95.

49. Talbot LA, Gaines JM, Huynh TN, Metter EJ: A home-based pedometerdriven walking program to increase physical activity in older adults with osteoarthritis of the knee: a preliminary study. J Am Geriatr Soc 2003, 51:387-392.

50. Wyatt HR, Peters JC, Reed GW, Grunwald GK, Barry M, Thompson H, Jones J, Hill JO: Using Electronic Step Counters to Increase Lifestyle Physical Activity: Colorado on the Move. J Phys Act Health 2004, 1:181-190.

51. Watson N, Milat AJ, Thomas M, Currie J: The feasibility and effectiveness of pram walking groups for postpartum women in western Sydney. Health promotion journal of Australia: official journal of Australian Association of Health Promotion Professionals 2005, 16:93-99.

52. Cox KL, Burke V, Beilin LJ, Derbyshire AJ, Grove JR, Blanksby BA, Puddey IB: Short and long-term adherence to swimming and walking programs in older women-the Sedentary Women Exercise Adherence Trial (SWEAT 2). Prev Med 2008, 46:511-517.

53. Jancey JM, Clarke A, Howat PA, Lee AH, Shilton T, Fisher J: A physical activity program to mobilize older people: a practical and sustainable approach. Gerontologist 2008, 48:251-257.

54. Merom D, Rissel C, Phongsavan P, Smith BJ, Van Kemenade C, Brown WJ, Bauman AE: Promoting walking with pedometers in the community: the step-by-step trial. Am J Prev Med 2007, 32:290-297.

55. Currie J, Develin E: Factors to consider when designing community pramwalking programs. American Journal of Health Education 2001, 32:48-51.

56. Engel $\mathrm{L}$, Lindner $\mathrm{H}$ : Impact of using a pedometer on time spent walking in older adults with type 2 diabetes. Diabetes Educ 2006, 32:98-107.

57. Assessing the sustainability of the 'Just Walk it' program: Is it effective and will it engance programm success. [http://www.transport.wa.gov.au/ conferences/walking/pdfs/B1.pdf].

58. Humpel N, Marshall AL, Iverson D, Leslie $\mathrm{E}$, Owen N: Trial of print and telephone delivered interventions to influence walking. Prev Med 2004, 39:635-641.
59. Shaw $G$, Alfonso H, Howat P, Corben $\mathrm{K}$ : Use of pedometers in a workplace physical activity program. Australasian Journal of Podiatric Medicine 2004.

60. Thomas L, Williams M: Promoting physical activity in the workplace: using pedometers to increase daily activity levels. Health promotion journal of Australia: official journal of Australian Association of Health Promotion Professionals 2006, 17:97-102.

61. Department NSWH: Walk it: active local parks: the effect of park modifications and promotion on physical activity participation: summary report. Book Walk it: active local parks: the effect of park modifications and promotion on physical activity participation: summary report City: NSW Health Department; 2002, ed.^eds.

62. Prestwich A, Perugini M, Hurling R: Can implementation intentions and text messages promote brisk walking? A randomized trial. Health Psychol 2010, 29:40-49.

63. Baker G, Gray SR, Wright A, Fitzsimons C, Nimmo M, Lowry R, Mutrie N: The effect of a pedometer-based community walking intervention "Walking for Wellbeing in the West" on physical activity levels and health outcomes: a 12-week randomized controlled trial. Int I Behav Nutr Phys Act 2008, 5:44.

64. Gilson N, McKenna J, Cooke C: Experiences of route and task-based walking in a university community: qualitative perspectives in a randomized control trial. J Phys Act Health 2008, 5(Suppl 1):S176-182

65. Lamb SE, Bartlett HP, Ashley A, Bird W: Can lay-led walking programmes increase physical activity in middle aged adults? A randomised controlled trial. J Epidemiol Community Health 2002, 56:246-252.

66. Darker CD, French DP, Eves FF, Sniehotta FF: An intervention to promote walking amongst the general population based on an 'extended' theory of planned behaviour: a waiting list randomised controlled trial. Psychol Health 2010, 25:71-88.

67. Baker G, Mutrie N, Lowry R: Using pedometers as motivational tools: Are goals set in steps more effective than goals set in minutes for increasing walking? International Journal of Health Promotion and Education 2008, 46:21-26.

68. Rowley C, Dixon L, Palk R: Promoting physical activity: walking programmes for mothers and children. Community Pract 2007, 80:28-32.

69. Nguyen MN, Gauvin L, Martineau I, Grignon R: Sustainability of the impact of a public health intervention: lessons learned from the laval walking clubs experience. Health Promot Pract 2005, 6:44-52.

70. Arbour KP, Martin Ginis KA: A randomised controlled trial of the effects of implementation intentions on women's walking behaviour. Psychol Health 2009, 24:49-65.

71. Culos-Reed SN, Stephenson L, Doyle-Baker PK, Dickinson JA: Mall walking as a physical activity option: results of a pilot project. Can J Aging 2008, 27:81-87.

72. Kolt GS, Oliver M, Schofield GM, Kerse N, Garrett N, Latham NK: An overview and process evaluation of TeleWalk: a telephone-based counseling intervention to encourage walking in older adults. Health Promot Int 2006, 21:201-208.

73. De Cocker KA, De Bourdeaudhuij IM, Brown WJ, Cardon GM: Effects of "10,000 steps Ghent": a whole-community intervention. Am J Prev Med 2007, 33:455-463.

74. Napolitano MA, Marcus BH: Targeting and tailoring physical activity information using print and information technologies. Exerc Sport Sci Rev 2002, 30:122-128.

75. Harris TJ, Carey IM, Victor CR, Adams R, Cook DG: Optimising recruitment into a study of physical activity in older people: a randomised controlled trial of different approaches. Age Ageing 2008, 37:659-665

76. Margitic S, Sevick MA, Miller M, Albright C, Banton J, Callahan K, Garcia M, Gibbons L, Levine BJ, Anderson R, Ettinger W: Challenges faced in recruiting patients from primary care practices into a physical activity intervention trial. Activity Counseling Trial Research Group. Prev Med 1999, 29:277-286.

77. Tai SS, lliffe S: Considerations for the design and analysis of experimental studies in physical activity and exercise promotion: advantages of the randomised controlled trial. Br J Sports Med 2000, 34:220-224.

78. Dawson J, Hillsdon M, Boller I, Foster C: Perceived barriers to walking in the neighbourhood environment and change in physical activity levels over 12 months. Br J Sports Med 2007, 41:562-568.

79. Klesges LM, Estabrooks PA, Dzewaltowski DA, Bull SS, Glasgow RE: Beginning with the application in mind: designing and planning health 
behavior change interventions to enhance dissemination. Ann Behav Med 2005, , 29 Suppl: 66-75.

80. Raynor HA, Osterholt KM, Hart CN, Jelalian E, Vivier P, Wing RR: Evaluation of active and passive recruitment methods used in randomized controlled trials targeting pediatric obesity. Int J Pediatr Obes 2009, 4:224-232.

\section{doi:10.1186/1479-5868-8-137}

Cite this article as: Foster et al:: Recruiting participants to walking intervention studies: a systematic review. International Journal of Behavioral Nutrition and Physical Activity 2011 8:137.

Submit your next manuscript to BioMed Central and take full advantage of:

- Convenient online submission

- Thorough peer review

- No space constraints or color figure charges

- Immediate publication on acceptance

- Inclusion in PubMed, CAS, Scopus and Google Scholar

- Research which is freely available for redistribution

Submit your manuscript at www.biomedcentral.com/submit
Ciomed Central 